\title{
Parameter Selection and Verification Techniques Based on Global Sensitivity Analysis Illustrated for an HIV Model
}

\author{
Mami T. Wentworth, Ralph C. Smith and H.T. Banks \\ Department of Mathematics \\ Center for Research in Scientific Computation \\ North Carolina State University \\ Raleigh, NC 27695
}

February 11, 2015

\begin{abstract}
We consider parameter selection and verification techniques for models having one or more parameters that are noninfluential in the sense that they minimally impact model outputs. We illustrate these techniques for a dynamic HIV model but note that the parameter selection and verification framework is applicable to a wide range of biological and physical models. To accommodate the nonlinear input to output relations, which are typical for such models, we focus on global sensitivity analysis techniques, including those based on partial correlations, Sobol indices based on second-order model representations, and Morris indices, as well as a parameter selection technique based on standard errors. A significant objective is to provide verification strategies to assess the accuracy of those techniques, which we illustrate in the context of the HIV model.
\end{abstract}

\section{Introduction}

Biological and physical models commonly have tens to hundreds of inputs - comprised of parameters, discretized spatially-varying coefficients, initial or boundary conditions, or exogenous forces - many of which have minimal influence on model responses. This necessitates the development of robust analysis techniques to establish subsets or subspaces of influential parameters or inputs. This challenge is exacerbated for models such as neutronics equations, which can have $10^{6}$ inputs, of which only 50-100 are considered influential. The need for robust parameter selection techniques is further motivated by the following objectives: (i) determine those inputs that can be uniquely estimated from measured data; (ii) establish the robustness or fragility of models with respect to certain parameter sets; (iii) simplify models by fixing insensitive inputs; and (iv) guide experimental design by ascertaining parameter subsets or subspaces that have the greatest impact on parameter or response sensitivity.

To establish notation and terminology, we consider the nonlinear input-output relation

$$
y=f(q)
$$

where $q=\left[q_{1}, \ldots, q_{p}\right]$ denotes the model inputs - e.g., parameters, initial or boundary conditions - and $f$ denotes the mathematical model. For this discussion, we consider real-valued responses $y \in \mathbb{R}^{1}$.

A significant goal of input or parameter selection techniques is to establish subsets or subspaces of inputs or parameters that can be uniquely identified from data or that strongly influence model responses. Such subsets can be characterized by the concepts of identifiable and influential parameter sets.

The concept of identifiability is classical and can be defined as follows. The parameters $q=\left[q_{1}, \ldots, q_{p}\right]$ are identifiable at $q^{*}$ if $f(q)=f\left(q^{*}\right)$ implies that $q=q^{*}$ for all admissible $q \in \mathbb{Q}$. The parameters $q$ are identifiable with respect to a space $\mathcal{I}(q)$, termed the identifiable subspace, if this holds for all $q^{*} \in I(q)$. 


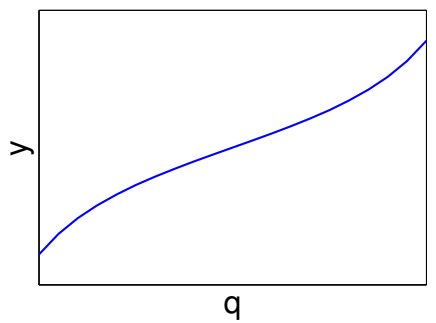

(a)

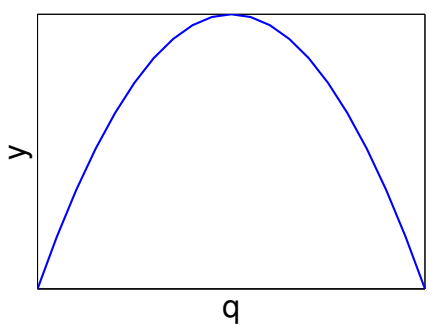

(b)

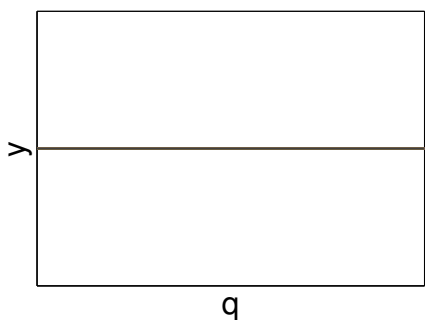

(c)

Figure 1: Illustration of $y=f(q)$ for (a) identifiable, (b) unidentifiable and (c) noninfluential parameters $q$.

Hence identifiable parameters can be uniquely determined from observations. An example of identifiable and nonidentifiable parameters are illustrated in Figure 1 (a) and (b).

Influential parameter spaces are sometimes defined differently in various disciplines. We define the parameter set $q=\left[q_{1}, \ldots, q_{p}\right]$ to be noninfluential on the space $\mathcal{N} \mathcal{I}(q)$ if $f(q)-f\left(q^{*}\right)<\varepsilon$ for all $q$ and $q^{*} \in \mathcal{N} \mathcal{I}(q)$. The space $\mathcal{I}(q)$ of influential parameters is defined to be the orthogonal component of $\mathcal{N} \mathcal{I}(q)$. Noninfluential parameters, like nonidentifiable parameters, can be fixed for model calibration and uncertainty propagation. Hence, the space of noninfluential parameters is a subspace of the space of nonidentifiable parameters. An example of a noninfluential parameter is illustrated in Figure 1 (c). Furthermore, parameter $q_{1}$ is more influential than parameter $q_{2}$ if changes in $q_{1}$ affect greater changes in $y$ than changes in $q_{2}$ do. See Figure 2 for an example of highly and minimally influential parameters. We will quantify the degree of influence using global sensitivity analysis.

For linearly parameterized problems $y=A q$, it is shown in Chapter 6 of [20] that deterministic and parametrized QR or SVD algorithms can be used to determine subspaces of influential parameters. For the nonlinearly parametrized problems, one typically resorts to global sensitivity analysis or active subspace techniques.

In this paper, we focus on global sensitivity analysis and subset selection based on standard errors to determine subsets $q^{s}=\left\{q_{1}^{s}, \ldots, q_{\tilde{p}}^{s}\right\} \subset q=\left\{q_{1}, \ldots, q_{p}\right\}$ of influential parameters. This differs from subspace selection techniques - typically based on QR or SVD algorithms with inputs randomly selected from the admissible input space - which can include linear combinations of inputs [2, 11, 20]. The comparison of active input subspaces with the subset established here for the HIV model constitutes future research.

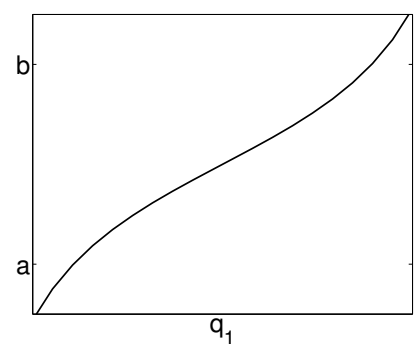

(a)

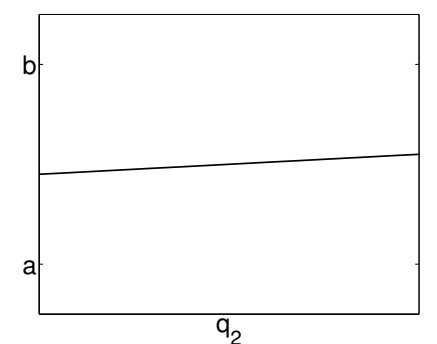

(b)

Figure 2: Illustration of influential parameters where $q_{1}$ is more influential than $q_{2}$.

\subsection{HIV Model, Inputs and Responses}

We illustrate the parameter selection and verification strategies in the context of an HIV model presented in [5] to understand mechanisms regarding the disease dynamics and to develop optimal treatment strategies. The model's predictive capability has been verified using data from patients who underwent a clinical study involving structured treatment interruptions. The system of ODE modeling the HIV disease in $[5$ is given by 


$$
\begin{aligned}
\dot{T_{1}} & =-d_{1} T_{1}-\left(1-\xi_{1}(t)\right) k_{1} V_{I} T_{1}-\gamma_{T} T_{1}+p_{T}\left(\frac{a_{T} V_{I}}{V_{I}+K_{V}}+a_{A}\right) T_{2} \\
\dot{T_{1}^{*}} & =\left(1-\xi_{1}(t)\right) k_{1} V_{I} T_{1}-\delta T_{1}^{*}-m E_{1} T_{1}^{*}-\gamma_{T} T_{1}^{*}+p_{T}\left(\frac{a_{T} V_{I}}{V_{I}+K_{V}}+a_{A}\right) T_{2}^{*} \\
\dot{T_{2}} & =\lambda_{T} \frac{K_{s}}{V_{I}+K_{s}}-\gamma_{T} T_{1}-d_{2} T_{2}-\left(1-f \xi_{1}(t)\right) k_{2} V_{I} T_{2}-\left(\frac{a_{T} V_{I}}{V_{I}+K_{V}}+a_{A}\right) T_{2} \\
\dot{T_{2}^{*}} & =\gamma_{T} T_{1}^{*}+\left(1-f \xi_{1}(t)\right) k_{2} V_{I} T_{2}-d_{2} T_{2}^{*}-\left(\frac{a_{T} V_{I}}{V_{I}+K_{V}}+a_{A}\right) T_{2}^{*} \\
\dot{V_{I}} & =\left(1-\xi_{2}(t)\right) 10^{3} N_{T} \delta T_{1}^{*}-c V_{I}-10^{3}\left[\left(1-\xi_{1}(t)\right) \rho_{1} k_{1} T_{1}+\left(1-f \xi_{1}(t)\right) \rho_{2} k_{2} T_{2}\right] V_{I} \\
\dot{V_{N I}} & =\xi_{2}(t) 10^{3} N_{T} \delta T_{1}^{*}-c V_{N I} \\
\dot{E_{1}} & =\lambda_{E}+\frac{b_{E_{1}} T_{1}^{*}}{T_{1}^{*}+K_{b 1}} E_{1}-\frac{d_{E} T_{1}^{*}}{T_{1}^{*}+K_{d}} E_{1}-\delta_{E 1} E_{1}-\gamma_{E} \frac{T_{1}+T_{1}^{*}}{T_{1}+T_{1}^{*}+K_{\gamma}} E_{1}+\frac{p_{E} a_{E} V_{I}}{V_{I}+K_{V}} E_{2} \\
\dot{E_{2}} & =\gamma_{E} \frac{T_{1}+T_{1}^{*}}{T_{1}+T_{1}^{*}+K_{\gamma}} E_{1}+\frac{b_{E 2} K_{b 2}}{E_{2}+K_{b 2}} E_{2}-\delta_{E 2} E_{2}-\frac{a_{E} V_{I}}{V_{I}+K_{V}} E_{2}
\end{aligned}
$$

with initial conditions $\left[T_{1}(0), T_{1}^{*}(0), T_{2}(0), T_{2}^{*}(0), V_{I}(0), V_{N I}(0), E_{1}(0), E_{2}(0)\right]$. Here, $T_{1}$ and $T_{1}^{*}$ respectively denote uninfected and infected activated (antigen-specific) CD4+ T-cells. Uninfected resting, i.e., not activated, CD4+ T-cells are denoted by $T_{2}$ and infected resting CD4+ T-cells are denoted by $T_{2}^{*}$. Infectious free virus is denoted by $V_{I}$; this is the virus that is capable of infecting other cells in the plasma. On the other hand, $V_{N I}$ denotes non-infectious free virus, which is yielded inactive by protease inhibitors. HIV-specific effector CD8+ T-cells are denoted by $E_{1}$ and HIV-specific memory CD8+ T-cells are denoted by $E_{2}$. The compartments of the model are depicted in Figure 3.

Several terms in the model (1) are based on the law of mass action, so that the rate of change in population size is proportional to the population size. The terms $-d_{1} T_{1}$ and $\gamma_{T} T_{1}$ in $\dot{T}_{1}$ are examples of mass action terms. Other terms are based on Michaelis-Menten kinetics, in which the rate saturates at a maximum. An example of this type is $\frac{a_{T} V_{I}}{V_{I}+K_{V}}$, which is the activation of infected HIV specific resting CD4+ T-cells with $a_{T}$ being the maximum activation rate. The term $\frac{\lambda_{T} K_{S}}{V_{I}+K_{S}}$ in the differential equation for $\dot{T}_{2}^{*}$ accounts for the source rate of naive CD4+ T-cells. In the equation for $\dot{E}_{1}, \frac{b_{E 1} T_{1}^{*}}{T_{1}^{*}+K_{b 1}} E_{1}$ and $-\frac{d_{E} T_{1}^{*}}{T_{1}^{*}+K_{d}} E_{1}$ respectively represent dynamic effects that activated, infected CD4+ T-cells have on the effector CD8+ T-cells when

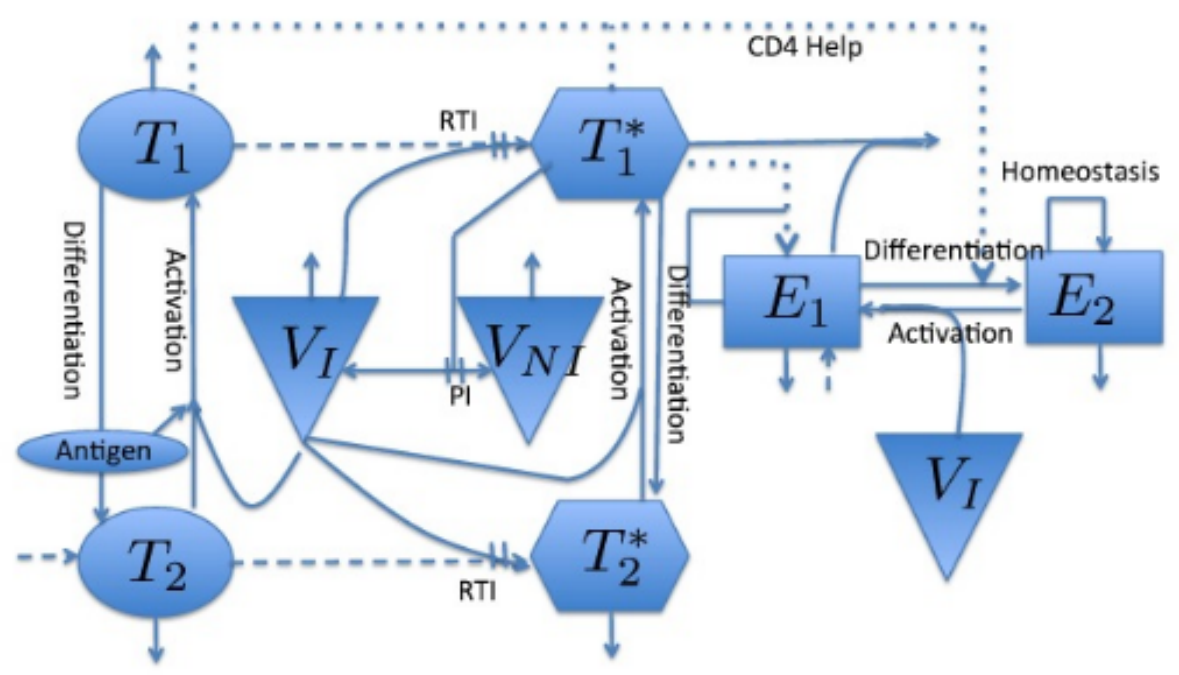

Figure 3: The compartments of HIV model (1). 


\begin{tabular}{|c|l|}
\hline Parameter & Explanation \\
\hline$\delta$ & Viral produced lysis rate of $T_{1}^{*}$ \\
$d_{2}$ & $T_{2}$ and $T_{2}^{*}$ natural death rate \\
$\delta_{E 2}$ & Death rate of $E_{2}$ \\
$m$ & Rate of removal by cell lysis of $T_{1}^{*}$ from the system by $E_{1}$ \\
$\gamma_{T}$ & Rate at which $T_{1}$ and $T_{1}^{*}$ differentiate into $T_{2}$ and $T_{2}^{*}$, respectively \\
$c$ & Natural clearance rate of $V_{I}$ and $V_{N I}$ \\
$\delta_{E 1}$ & Constant death rate of $E_{1}$ \\
$\gamma_{E}$ & Source term for $E_{1}$ \\
$k_{2}$ & Production rate of $T_{2}^{*}$ due to encounters between $T_{2}$ and $V_{I}$ that is less than $k_{1}$ \\
$\rho_{1}$ & Rate of removal of $V_{I}$ I through successful infection of $T_{1}$ \\
$\rho_{2}$ & Rate of removal of $V_{I}$ through successful infection of $T_{2}$ \\
$d_{1}$ & Natural death rate of $T_{1}$ \\
$\epsilon_{2}$ & Relative effectiveness of protease inhibitor $($ PI) \\
$a_{A}$ & Activation rate of $T_{2}$ and ${ }_{2}^{*}$ by non-HIV antigen \\
$\epsilon_{1}$ & Relative effectiveness of reverse transcriptase inhibitor $(\mathrm{RTI})$ \\
$p_{T}$ & Net proliferation of $T_{1}$ and $T_{1}^{*}$ due to clonal expansion and programmed contraction \\
$p_{E}$ & Net proliferation of $E_{1}$ due to clonal expansion and programmed contraction \\
$k_{1}$ & Production rate of $T_{1}^{*}$ from encounters between $T_{1}$ and $V_{I}$ \\
$N_{T}$ & Number of RNA copies produced during the process of $T_{1}^{*}$ lysis \\
$a_{T}$ & Maximum activation rate of $T_{2}$ and $T_{2}^{*}$ \\
$f$ & Efficacy of treatment $0 \leq f \leq 1$ \\
$\lambda_{E}$ & Constant differentiation of $E_{2}$ into $E_{1}$ \\
$K_{V}$ & Half-saturation constant of virus \\
\hline
\end{tabular}

Table 1: Description of parameters in the model (1).

$K_{b 1}<K_{d}$ and $b_{E 1}<b_{E}$. Also in this differential equation, $\frac{p_{E} a_{E} V_{I}}{V_{I}+K_{V}} E_{2}$ represents the activation of memory CD8+ T-cells into effector CD8+ T-cells. In the differential equation for $\dot{E}_{2}, \frac{E_{1}\left(T_{1}+T_{1}^{*}\right)}{T_{1}+T_{1}^{*}+K_{\gamma}}$ has an essential role that activated CD4+ T-cells play in the generation of memory CD8+ T-cells, whereas $\frac{b_{E 2} K_{b 2} E_{2}}{E_{2}+K_{b 2}}$ and $\delta_{E 2} E_{2}$ are homeostatic regulation terms in $E_{2}$.

The parameters in the HIV model (1) are described in Table 1 and nominal values for parameters and initial conditions reported in [3] are compiled in Table 2. The functions $\xi_{1}=\epsilon_{1} u(t)$ and $\xi_{2}=\epsilon_{2} u(t)$ represent the impact of the treatment. Here, $\epsilon_{1}$ is the effectiveness of the reverse transcriptase inhibitor (RTI), whereas $\epsilon_{2}$ is the effectiveness of the protease inhibitor (PI). Also, $u(t)$ is the HAART drug level, where $u(t)=1$ when the patient is on treatment, and $u(t)=0$ when the patient is off treatment. Parameters such as $\epsilon_{1}$ and $\epsilon_{2}$, along with many others, can not be directly measured and hence must be estimated through a fit to data.

Among these parameters, however, some do not influence model outputs. These parameters must be identified via parameter selection prior to parameter estimation. Isolating these noninfluential parameters allows us to reduce the parameter dimensions for model calibration and focus on estimating those parameters that can be uniquely determined from the data.

Based on results from [3], we focus on the 15 parameters and initial conditions

$$
q=\left[\lambda_{T}, d_{1}, \epsilon_{1}, k_{1}, a_{T}, \epsilon_{2}, N_{T}, b_{E 2}, a_{E}, p_{E}, a_{A}, p_{T}, T_{1}(0), T_{1}^{*}(0), T_{2}(0)\right]
$$

whose values tend to be patient specific. Here, the input dimensions is $p=15$. The associated random variable, considered for global sensitivity analysis, is denoted by $Q$. Also, we denoted the admissible input space of biologically feasible parameters and initial conditions by $\mathbb{Q}$. The lower and upper bounds for each parameter, where $q_{i} \in\left[\ell b_{i}, u b_{i}\right]$, is summarized in Table 3. For more details of the terms and parameters, see 3 , 5].

As detailed in [5], data was collected from patients, in a clinical trial, who underwent anti-retroviral therapy (ART) and had at least one ART interruption. The total CD4+ T-cell count/micro L-blood $\left(T_{1}+\right.$ 


\begin{tabular}{|llll|}
\hline$\lambda_{T}=3.2543$ & $d_{1}=0.1317$ & $\epsilon_{1}=0.5241$ & $k_{1}=4.8200 \mathrm{e}-5$ \\
$a_{T}=2.3198 \mathrm{e}-4$ & $\epsilon_{2}=0.7149$ & $N_{T}=79.26$ & $b_{E 2}=0.34554$ \\
$a_{E}=1.5332 \mathrm{e}-2$ & $p_{E}=1.0294$ & $a_{A}=8.07 \mathrm{e}-5$ & $p_{T}=5.531$ \\
$\gamma_{T}=3.792 \mathrm{e}-4$ & $d_{2}=3.096 \mathrm{e}-3$ & $f=0.5068$ & $k_{2}=2.005 \mathrm{e}-9$ \\
$\delta=0.2095$ & $m=1.127 \mathrm{e}-3$ & $c=5.818$ & $\lambda_{E}=9.9930 \mathrm{e}-4$ \\
$b_{E 1}=3.885 \mathrm{e}-2$ & $K_{b 1}=2.488 \mathrm{e}-2$ & $d_{E}=6.278 \mathrm{e}-2$ & $K_{d}=0.12$ \\
$\delta_{E 1}=5.967 \mathrm{e}-2$ & $K_{b 2}=86.97$ & $\gamma_{E}=5.154 \mathrm{e}-4$ & $K_{\gamma}=1.357$ \\
$K_{V}=14.79$ & $\delta_{E 2}=1.450 \mathrm{e}-3$ & $K_{s}=2.789 \mathrm{e}+4$ & $T_{1}(0)=12.135$ \\
$T_{1}^{*}(0)=5.8604 \mathrm{e}-4$ & $T_{2}(0)=823.59$ & $T_{2}^{*}(0)=7.521 \mathrm{e}-3$ & $V_{I}(0)=3.571 \mathrm{e}+3$ \\
$V_{N I}(0)=3.571 \mathrm{e}+3$ & $E_{1}(0)=6.821 \mathrm{e}-2$ & $E_{2}(0)=0.6909$ & \\
\hline
\end{tabular}

Table 2: Nominal values of parameters and initial conditions from [3].

$\left.T_{1}^{*}+T_{2}+T_{2}^{*}\right)$ as well as total RNA copies/mL-plasma $\left(V_{I}+V_{N I}\right)$ were recorded during this process.

For global sensitivity analysis, we require a scaler response. At the same time, we are interested in how parameters affect the model output for the feasible input as well for the entire duration of therapy. For these reasons, we choose our scalar model response to be

$$
f(q)=\int_{0}^{1500} T_{1}(t ; q)+T_{1}^{*}(t ; q)+T_{2}(t ; q)+T_{2}^{*}(t ; q) d t+\int_{0}^{1500} V_{I}(t ; q)+V_{N I}(t ; q) d t .
$$

To test the parameter selection techniques, we generate synthetic data using the mean values from the model calibration performed in [5], which are summarized in Table 2. The model is solved numerically using ode15s in MATLAB.

\subsection{Previous Work and Paper Organization}

Whereas global sensitivity analysis techniques for parameter selection have not previously been investigated for this dynamic HIV model, certain techniques have been used to analyze other biological models.

Readers are referred to [10] for a case study illustrating the use of sensitivity analysis for a rice model, and 13, 23. for examples of parameter selection in computational and systems biology. The subset selection developed in [4, 6, 9] is applied to the HIV model (1) in [3] and we compare our sensitivity-based parameter subsets to those of 3 in Section 4 .

In Section 2, we illustrate the difference between local and global sensitivity analysis using a simple portfolio model. In Section 3, we discuss four different techniques for parameter selection. We start with Partial Correlation [1, which quantifies the linear effects of parameters on the model response. Secondly, we discuss Sobol indices, which are variance-based methods based on a second-order Sobol decomposition. For the HIV example, we discuss the limited accuracy of this decomposition and its affect on parameter selection. Thirdly, we summarize Morris indices using a screening method that ranks parameters in the order of importance. Finally, we discuss the parameter subset selection algorithm discussed in [3]. In Section 4, we present our results of applying parameter selection techniques to the HIV model. We interpret the sensitivity indices from each method and provide a comparison for identifying influential parameters. We present verification techniques to illustrate that non-influential parameters should not affect the model

\begin{tabular}{|c|cccccccc|}
\hline & $\lambda_{T}$ & $d_{1}$ & $\epsilon_{1}$ & $k_{1}$ & $a_{T}$ & $\epsilon_{2}$ & $N_{T}$ & $b_{E 2}$ \\
\hline$\ell b_{i}$ & 3.1 & 0.11 & 0.43 & $4.0 \mathrm{e}-5$ & $2.0 \mathrm{e}-4$ & 0.63 & 65 & 0.28 \\
$u b_{i}$ & 3.5 & 0.15 & 0.6 & $5.5 \mathrm{e}-5$ & $2.7 \mathrm{e}-4$ & 0.78 & 85 & 0.45 \\
\hline \hline & $a_{E}$ & $p_{E}$ & $a_{A}$ & $p_{T}$ & $T_{1}(0)$ & $T_{1}^{*}(0)$ & $T_{2}(0)$ & \\
\hline$\ell b_{i}$ & $1.40 \mathrm{e}-3$ & 0.85 & $6.5 \mathrm{e}-5$ & 5 & 10.5 & $5.0 \mathrm{e}-4$ & 720 & \\
$u b_{i}$ & $1.75 \mathrm{e}-3$ & 1.3 & $9.0 \mathrm{e}-5$ & 6.5 & 13.5 & $7.0 \mathrm{e}-4$ & 950 & \\
\hline
\end{tabular}

Table 3: The lower and upper bounds of parameters, $q_{i} \in\left[\ell b_{i}, u b_{i}\right]$, for $i=1, \ldots, 15$. 
output when fixed at nominal values. Finally, we provide comprehensive implications of parameter selection techniques on the HIV model.

\section{Global Sensitivity Motivation}

There are two types of sensitivity analysis: local versus global. In literature, sensitivity analysis often refers to the local sensitivity analysis, which is typically computed by evaluating the derivative of the response with respect to inputs at nominal input values. On the other hand, global sensitivity analysis considers the effect of parameters over the entire range of input values. Global sensitivity analysis is also used to ascertain how uncertainty in model outputs is apportioned to uncertainties in model inputs; see [17, 19, 20, 21] for details.

We note that global sensitivity techniques rank the relative impact of influential inputs or parameters. Further tests are required to establish that least influential parameters are non-influential in the sense defined in Section 1 .

To illustrate the difference between local sensitivity analysis and global sensitivity analysis, we begin by considering the linear portfolio model

$$
Y=c_{1} Q_{1}+c_{2} Q_{2}
$$

considered in 19, 20. Here, the random variable $Y$ is the return for the investment and $Q_{1} \sim N\left(0, \sigma_{1}^{2}\right)$ and $Q_{2} \sim N\left(0, \sigma_{2}^{2}\right)$ represent hedged portfolios, where $c_{1}$ and $c_{2}$ are the amounts invested in each portfolio. In this example, we take $c_{1}=2, c_{2}=1, \sigma_{1}=1$ and $\sigma_{2}=3$. The fact that $\sigma_{2}>\sigma_{1}$ implies that the second portfolio is more volatile than the first. The scatterplots of 1000 joint realizations of $q_{1}, q_{2}$ and $y$ in Figure 4 indicate that $Q_{2}$ has more influence on $Y$ than $Q_{1}$. Hence, globally, $Y$ is more sensitive to $Q_{2}$ than $Q_{1}$.

However, the local sensitivity $s_{i}=\frac{\partial Y}{\partial Q_{i}}$ for $i=1,2$ yields $s_{1}=2$ and $s_{2}=1$, indicating that $q_{1}$ is more sensitive. This reflects the amounts invested in the two portfolio rather than the effects of their volatility of the return. Hence the local sensitivity does not incorporate the nonlinear uncertainty structure over the global admissible parameter space nor the effect of parameter variability on the response.

In our HIV example, we are interested in how parameters affect the model response in the entire parameter space, rather than at some nominal parameter values. For this reason, we use global sensitivity analysis as a parameter selection technique and isolate influential parameters from noninfluential parameters. In the next section, we discuss three methods of parameter selection based on global sensitivity analysis and one method based on standard errors.

\section{Parameter Selection Methods}

The first of the four parameter selection methods that we discuss is termed the Partial Correlation, or Pearson's Correlations. This method quantifies the linear effect of parameters on the model response. Secondly, we detail the use of Sobol indices based on a variance-based, second-order Sobol decomposition. As an initial step, we examine and verify the accuracy of the second-order expansions. Thirdly, we consider the Morris screening method. We note that this method provides a mechanism of ranking parameters but does
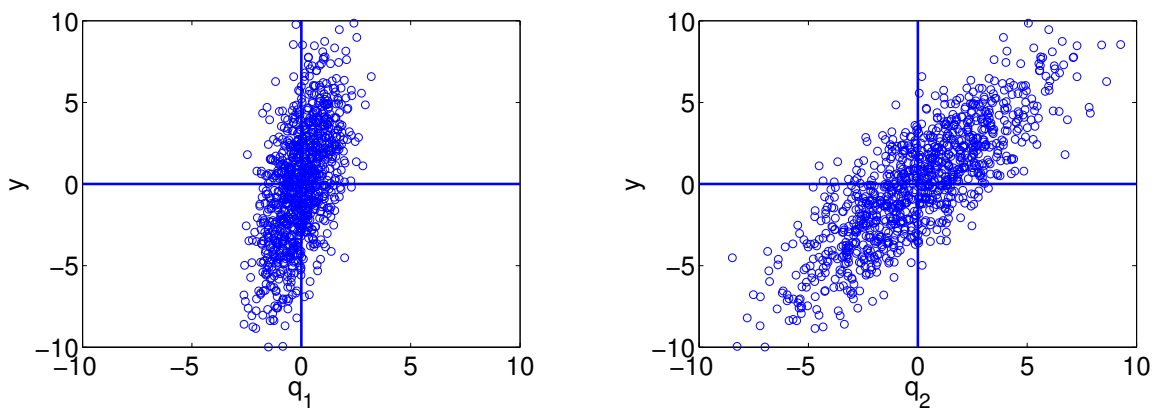

Figure 4: Scatterplots of 1000 joint realizations of $y$ versus (a) $q_{1}$ and (b) $q_{2}$. 
not necessarily quantify their relative importance. Finally, we summarize the parameter subset selection detailed in [3]. This method quantifies the importance of parameters by comparing a dimensionless ratio of standard error and mean for each parameter.

\subsection{Partial Correlation}

We begin by computing partial correlations as detailed in [1]. For two random variables $X$ and $Y$, the covariance is given by

$$
\operatorname{cov}(X, Y)=\mathbb{E}[(x-\mathbb{E}(X))(Y-\mathbb{E}(Y))]=\mathbb{E}(X Y)-\mathbb{E}(X) \mathbb{E}(Y)
$$

The partial correlation is then given by

$$
\rho_{X Y}=\frac{\operatorname{cov}(X, Y)}{\sigma_{X} \sigma_{Y}} .
$$

The partial correlation quantifies the degree to which two random variables are correlated. For example, $\rho_{X Y}=0$ indicates that $X$ and $Y$ are not correlated. We note that it does not imply that the two random variables are independent since (4) only quantifies linear dependencies between parameters. On the other hand, $\rho_{X Y}= \pm 1$ indicates a linear algebraic relation between the variables, in which case they are not jointly identifiable. Values greater than 0.5 generally indicate significant correlations. However, one must study the parameters with partial correlation values less than 0.5 for possible confounding factors or nonlinearities before determining insignificant.

For the HIV example, $X=Q_{i}$ denotes the random variable for the $i^{\text {th }}$ parameter, and $Y$ is the random variable representing the model response. The partial correlation then quantifies the degree of linear correlation between a parameter $q_{i}$ and model response $y$. We compute the correlation

$$
\rho_{q_{i} y}=\frac{\sum_{j}\left(\left(q_{i}\right)_{j}-\bar{q}_{i}\right)\left(y_{j}-\bar{y}\right)}{\sqrt{\sum_{j}\left(\left(q_{i}\right)_{j}-\bar{q}_{i}\right)^{2} \sum_{k}\left(y_{k}-\bar{y}\right)^{2}}}
$$

where $\bar{q}_{i}$ and $\bar{y}$ are the means of $q_{i}$ and $y$, respectively. The number of function evaluations required to compute the partial correlation using $M$ Monte Carlo evaluations for $p$ parameters is then $M \times p$.

For this method, variables with large partial correlations are considered more influential on the response than those yielding small values of $\rho_{Q_{i} Y_{i}}$. For the portfolio model (3), this would reflect the results shown in Figure 4, which indicate that $Q_{2}$ is more influential than $Q_{1}$.

\subsubsection{Partial Correlation Results}

The partial correlation values are computed for the model (1) using $M=2000$ function evaluations per parameter. The result is plotted in Figure 5 to provide visual comparison for the overall input-output correlation. Since we are interested in the magnitude of correlation values, the negative correlation values are also shown in the positive direction.

The result indicates that $N_{T}$ is most correlated to the model response. Also, $p_{T}$ and $k_{1}$ are more correlated to the model response than other variables. On the other hand, two of the initial conditions, $T_{1}(0)$ and $T_{1}^{*}(0)$ are not correlated to the model response, implying that they have minimum influence.

\subsection{Sobol Indices}

To construct Sobol indices, we assume that parameters have been mapped to $[0,1]$ and that $q \sim \mathcal{U}[0,1]^{p}$. Details regarding the construction of Sobol indices for general densities are provided in [20]. 


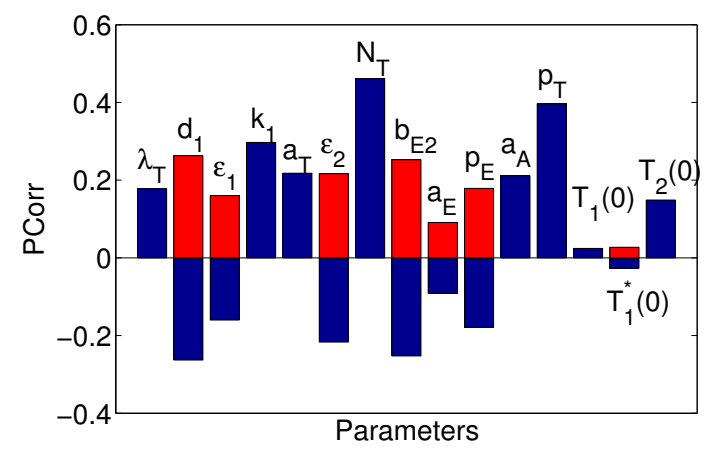

Figure 5: Partial correlation of the scalar response to the input parameters.

\subsubsection{Sobol Decomposition}

Sobol indices are based on a second-order High Dimensional Model Representation (HDMR) or Sobol representation

$$
f(q) \approx f_{0}+\sum_{i=1}^{p} f_{i}\left(q_{i}\right)+\sum_{1 \leq i<j \leq p} f_{i j}\left(q_{i}, q_{j}\right) .
$$

Since the representation (5) is not unique, additional conditions are imposed to ensure the uniqueness of component functions $f_{i}$ and $f_{i j}$. As detailed in [14, 16, 20, 21, each component function is uniquely specified by minimizing the functional

$$
\left.\mathcal{J}=\int_{\Gamma^{p}}\left[f(q)-\left(f_{0}+\sum_{i}^{p} f_{i}\left(q_{i}\right)+\cdots+\sum_{i_{1}<\cdots<i_{s}} f_{i_{1}, \cdots, i_{s}} q_{i_{1}}, \cdots, q_{i_{s}}\right)\right)\right]^{2} d q
$$

subject to

$$
\int_{\Gamma} f_{i_{1}, \ldots, i_{s}}\left(q_{i_{1}}, \ldots, q_{i_{s}}\right) d q_{i_{k}}=0
$$

for $k=1, \ldots, s$ and $s=1, \ldots, p$.

The component functions are given by

$$
\begin{aligned}
f_{i} & =\int_{\Gamma^{p-1}} f(q) d q_{\sim i} \\
f_{i j} & =\int_{\Gamma^{p-2}} f(q) d q_{\sim i, j}
\end{aligned}
$$

where $\Gamma^{k}=[0,1]^{k}$ for a positive integer $k$ and the notation $d q_{\sim i}$ denotes $d q_{1}, \ldots, d q_{i-1}, d q_{i+1}, \ldots, d q_{p}$.

The variance-based method employs the expansion (5) to quantify the contribution of each parameter to the variance of response. As detailed in [20, the total variance of response $\mathrm{Y}$ is given by

$$
D=\operatorname{var}(Y)=\int_{\Gamma} f^{2}(q) d q-f_{0}^{2}
$$

where $f_{0}$ is the mean response given by

$$
f_{0}=\int_{\Gamma} f(q) d q
$$

The total variance can be expressed as a sum of variances due to first-order and second-order parameter interactions by expressing $D$ as

$$
D=\sum_{i=1}^{p} D_{i}+\sum_{1 \leq i<j \leq p} D_{i j}
$$


where

$$
\begin{aligned}
D_{i} & =\int_{\Gamma} f_{i}^{2}\left(q_{i}\right) d q_{i} \\
D_{i j} & =\int_{\Gamma^{2}} f_{i j}^{2}\left(q_{i}, q_{j}\right) d q_{i} d q_{j} .
\end{aligned}
$$

The Sobol indices are then defined to be

$$
S_{i}=\frac{D_{i}}{D}, S_{i j}=\frac{D_{i j}}{D}, i, j,=1, \ldots, p .
$$

Here $S_{i}$ are often called the importance measures or first-order sensitivity indices, and they measure the contribution of the parameter $q_{i}$ on the response variance. A large value of $S_{i}$ implies stronger influence of parameter $q_{i}$ on the response variance. Similarly, $S_{i j}$ measures the contribution of parameter interactions between $q_{i}$ and $q_{j}$ on the response variance. Since the computation of first- and second- order sensitivity indices requires $p+\frac{p(p-1)}{2}$ model responses, we instead consider the total sensitivity indices

$$
S_{T_{i}}=S_{i}+\sum_{j=1}^{p} S_{i j}
$$

which quantify the total effect of the parameter $q_{i}$ on the response [20].

\subsubsection{Statistical Interpretation}

The Sobol indices, along with the expansion terms and partial variances, have expectation or variance interpretations. Let

$$
\begin{aligned}
\mathbb{E}\left(Y \mid q_{i}\right) & =\int_{\Gamma^{p-1}} f(q) d q_{\sim i} \\
\mathbb{E}\left(Y \mid q_{i}, q_{j}\right) & =\int_{\Gamma^{p-2}} f(q) d q_{\sim\{i j\}}
\end{aligned}
$$

denote the expected response when $q_{i}$ and $q_{i}, q_{j}$ are fixed. The component functions are

$$
\begin{aligned}
& f_{0}=\mathbb{E}(Y) \\
& f_{i}\left(q_{i}\right)=\mathbb{E}\left(Y \mid q_{i}\right)-f_{0} \\
& f_{i j}\left(q_{i}, q_{j}\right)=\mathbb{E}\left(Y \mid q_{i}, q_{j}\right)-f_{i}\left(q_{i}\right)-f_{j}\left(q_{j}\right)-f_{0} .
\end{aligned}
$$

As detailed in [20],

$$
D_{i}=\operatorname{var}\left[\mathbb{E}\left(Y \mid q_{i}\right)\right]
$$

and hence

$$
S_{i}=\frac{\operatorname{var}\left[\mathbb{E}\left(Y \mid q_{i}\right)\right]}{\operatorname{var}(Y)}
$$

Similarly, using the equality

$$
D_{i j}=\operatorname{var}\left[\mathbb{E}\left(Y \mid q_{i}, q_{j}\right)\right]-\operatorname{var}\left[\mathbb{E}\left(Y \mid q_{i}\right)\right]-\operatorname{var}\left[\mathbb{E}\left(Y \mid q_{j}\right)\right],
$$

the total sensitivity index has the variance interpretation

$$
S_{T i}=1-\frac{\operatorname{var}\left[\mathbb{E}\left(Y \mid q_{\sim i}\right)\right]}{\operatorname{var}(Y)}=\frac{\mathbb{E}\left[\operatorname{var}\left(Y \mid q_{\sim i}\right)\right]}{\operatorname{var}(Y)} .
$$

The interpretation of $\mathbb{E}\left(Y \mid q_{i}\right)$ and $\operatorname{var}\left[\mathbb{E}\left(Y \mid q_{i}\right)\right]$ is illustrated in Figure 6 from the portfolio example in Section 22 see also Chapter 15 of [20. The conditional expectations for fixed $q_{1}$ and $q_{2}$ are the average values of $Y$ along vertical slices. Again, we see that mean of response for fixed values of $q_{2}$ has more variance than that for fixed values of $q_{1}$. 

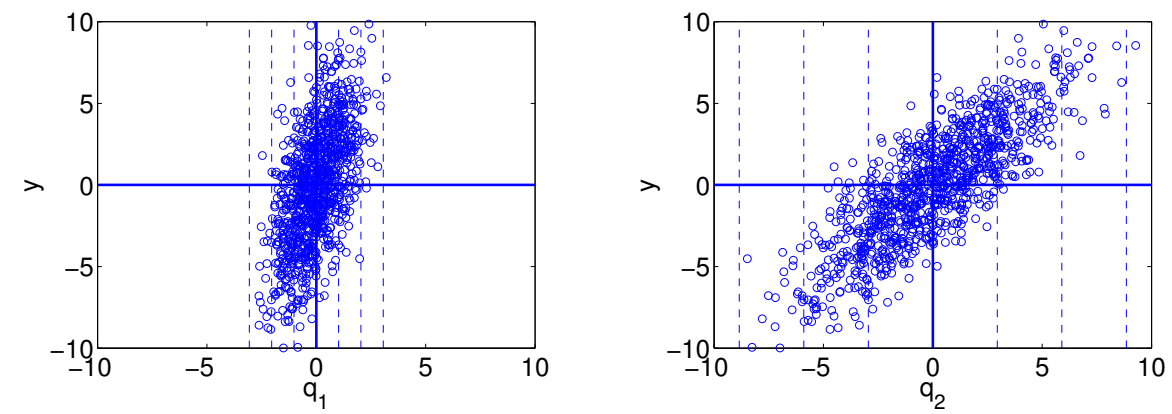

Figure 6: Response for fixed values of (a) $q_{1}$ and (b) $q_{2}$ illustrating $\mathbb{E}\left(Y \mid q_{i}\right)$ and $\operatorname{var}\left[\mathbb{E}\left(Y \mid q_{i}\right)\right]$.

\subsubsection{Sobol Indices Algorithm}

Since the computation of the Sobol indices requires high-dimensional integration, the indices are approximated numerically. If one uses $M$ Monte Carlo evaluations to approximate the mean $\mathbb{E}\left(Y \mid q_{i}\right)$ and repeats the procedure $M$ times to approximate the variance $\operatorname{var}\left[\mathbb{E}\left(Y \mid q_{i}\right)\right]$, a total of $M^{2}$ evaluations will be required to evaluate a single index. The total number of function evaluations required is $M^{2} p$, which is computationally prohibitive for a large parameter dimensions $p$. This motivated the author of [17] to provide a more efficient algorithm to compute Sobol indices that reduces the required evaluations to $M(p+2)$, based on Sobol's original approach in [21]. The algorithm was further improved by the authors of [18, 22] and is summarized here.

\section{Algorithm}

1. Create two sample matrices $A$ and $B$

$$
A=\left[\begin{array}{ccccc}
q_{1}^{1} & \ldots & q_{i}^{1} & \ldots & q_{p}^{1} \\
\vdots & & & & \vdots \\
q_{1}^{M} & \ldots & q_{i}^{M} & \ldots & q_{p}^{M}
\end{array}\right] \text {, and } B=\left[\begin{array}{ccccc}
\hat{q}_{1}^{1} & \ldots & \hat{q}_{i}^{1} & \ldots & \hat{q}_{p}^{1} \\
\vdots & & & & \vdots \\
\hat{q}_{1}^{M} & \ldots & \hat{q}_{i}^{M} & \ldots & \hat{q}_{p}^{M}
\end{array}\right]
$$

The entries $q_{i}^{j}$ and $\hat{q}_{i}^{j}$ are quasi-random numbers drawn from the respective densities.

2. Create $A_{B}^{(i)}$

$$
A_{B}^{(i)}=\left[\begin{array}{ccccc}
q_{1}^{1} & \ldots & \hat{q}_{i}^{1} & \ldots & q_{p}^{1} \\
\vdots & & & & \vdots \\
q_{1}^{M} & \ldots & \hat{q}_{i}^{M} & \ldots & q_{p}^{M}
\end{array}\right]
$$

which is the matrix $A$ except that $i^{\text {th }}$ column is taken from $B$. Similarly, create $B_{A}^{(i)}$.

3. Create $C$ which is the matrix $B$ appended to matrix $A$ such that

$$
C=\left[\begin{array}{c}
A \\
- \\
B
\end{array}\right]
$$

The rows of $C$ are linearly independent, and this matrix $C$ is used when estimating the total variance.

4. Compute column vectors $f(A), f(B), f\left(A_{B}^{(i)}\right)$ and $f\left(B_{A}^{(i)}\right)$ by evaluating the model at input values from the rows of matrices $A, B, A_{B}^{(i)}$ and $B_{A}^{(i)}$. Let $f(A)_{j}$ denote the output computed from the $j^{\text {th }}$ row of $A$. The computation of $f(A)$ and $f(B)$ requires $2 M$ model evaluations, whereas the evaluation of $f\left(A_{B}^{(i)}\right)$ and $f\left(B_{A}^{(i)}\right)$ for $i=1, \ldots, p$ requires $2 M p$ evaluations. The total number of model evaluations is $2 M(1+p)$. 
5. Estimate the Sobol indices. The first-order Sobol indices are approximated by

$$
S_{i}=\frac{\frac{1}{M} \sum_{j=1}^{M}\left[f(A)_{j} f\left(B_{A}^{(i)}\right)_{j}-f(A)_{j} f(B)_{j}\right]}{\frac{1}{2 M} \sum_{j=1}^{2 M} f(C)_{j} f(C)_{j}-\langle f(C)\rangle^{2}}
$$

and the total Sobol indices are approximated by

$$
S_{T i}=\frac{\frac{1}{2 M} \sum_{j=1}^{M}\left[f(A)_{j}-f\left(A_{B}^{(i)}\right)_{j}\right]^{2}}{\frac{1}{2 M} \sum_{j=1}^{2 M} f(C)_{j} f(C)_{j}-\langle f(C)\rangle^{2}} .
$$

In the last step, variances are approximated using Monte Carlo approximation. The denominator in (9) and 10 is the approximation for the total variance with $\mathbb{E}\left(Y^{2}\right) \approx \frac{1}{2 M} \sum_{j=1}^{2 M} f(C)_{j} f(C)_{j}$ and $(\mathbb{E}(Y))^{2}=$

$\langle f(C)\rangle^{2}$. In $\left[97\right.$, the term $\frac{1}{M} \sum_{j=1}^{M} f(A)_{j} f\left(B_{A}^{(i)}\right)_{j}$ approximates $\mathbb{E}\left(\mathbb{E}\left(Y \mid q_{i}\right)\right)^{2}$. In essence, we are taking the mean of responses when all input parameters are varied except $q_{i}$. The effect of $q_{i}$ is fixed since the $i^{t h}$ column is the same in both $A$ and $B_{A}^{(i)}$.

The second term in (9),

$$
\frac{1}{M} \sum_{j=1}^{M} f(A)_{j} f(B)_{j},
$$

represents the squared mean, $f_{0}^{2}$, using the identify

$$
f_{0}^{2}=\int_{\Gamma^{2}} f(x) f\left(x^{\prime}\right) d x d x^{\prime}
$$

This approximation is shown in 22] to reduce the loss of accuracy when computing $D$, compared to

$$
f_{0}^{2} \approx\left(\frac{1}{M} \sum_{j=1}^{M} f(A)_{j}\right)\left(\frac{1}{M} \sum_{j=1}^{M} f(B)_{j}\right),
$$

which is used in the previous versions of the algorithm.

The computation of $S_{T i}$ follows from the derivations in [12], which uses the approximation

$$
\mathbb{E}\left[\operatorname{var}\left(Y \mid q_{\sim i}\right)\right] \approx \frac{1}{2 M} \sum_{j=1}^{M}\left[f(A)_{j}-f\left(A_{B}^{(i)}\right)_{j}\right]^{2}
$$

instead of the approximation

$$
\operatorname{var}\left[\mathbb{E}\left(Y \mid q_{\sim i}\right)\right] \approx \frac{1}{M} \sum_{j=1}^{M} f(A)_{j} f\left(A_{B}^{(i)}\right)_{j}-f_{0}^{2}
$$

in (8). The comparison of different versions of the algorithm can be found in [18].

\subsubsection{Sobol Indices Results}

The Sobol indices for the 15 parameters in (2) are plotted in Figure 7 It is clear that $N_{T}$ has the largest $S_{T i}$ value, indicating that $N_{T}$ affects the model output the most. We note that $p_{T}$ is also almost as significant. On the other hand, $T_{1}^{*}(0)$ affects the output the least and $a_{E}$ and $T_{1}(0)$ are also very insignificant. 


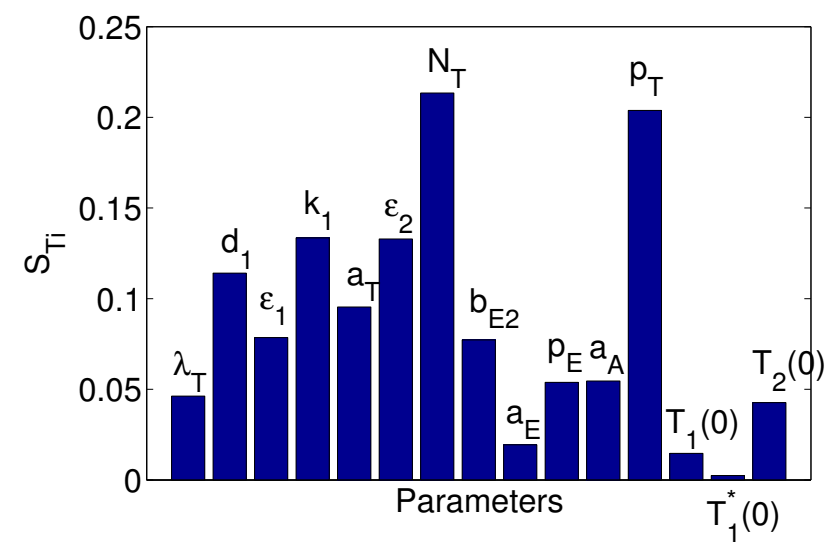

Figure 7: Sobol indices $S_{T i}$ for 15 parameters.

\subsubsection{Verification of the Sobol Decomposition}

Since the accuracy of the Sobol indices depends on the accuracy of the approximated second-order Sobol representation, we test whether the function is accurately approximated by the second-order Sobol decomposition.

To ensure that we can adequately approximate the integrals, we consider four parameters $q=\left[\lambda_{T}, d_{1}, \epsilon_{1}, k_{1}\right]$ with values in the 4 -D hypercube $[3.1,3.5] \times[0.11,0.15] \times[0.43,0.60] \times[4 \mathrm{e}-5,5.5 \mathrm{e}-5]$. We compute the model response using $n=41$ equally-spaced quadrature points in each dimension to evaluate the integrals (6) and (7). The function is expanded with a zero-th, first, second, third and fourth order component functions so that

$$
f=f_{0}+\sum_{i} f_{i}\left(q_{i}\right)+\sum_{i<j} f_{i j}\left(q_{i}, q_{j}\right)+\sum_{i<j<k} f_{i j k}\left(q_{i}, q_{j}, q_{k}\right)+\sum_{i<j<k<h} f_{i j k h}\left(q_{i}, q_{j}, q_{k}, q_{h}\right) .
$$

In Figure 8, we plot the model response along with first- and second-order approximations, where the fixed parameter values are taken to be $\lambda_{T}=3.19, \epsilon_{1}=0.119, d_{1}=0.46825, k_{1}=4.3375 \mathrm{e}-5$. The model response is represented by the blue solid line, while the first-order approximation and the second-order approximation are represented by dashed-dot black and by dashed red, respectively.

We note that both the first- and second-order approximations smooth out the jumps in the model
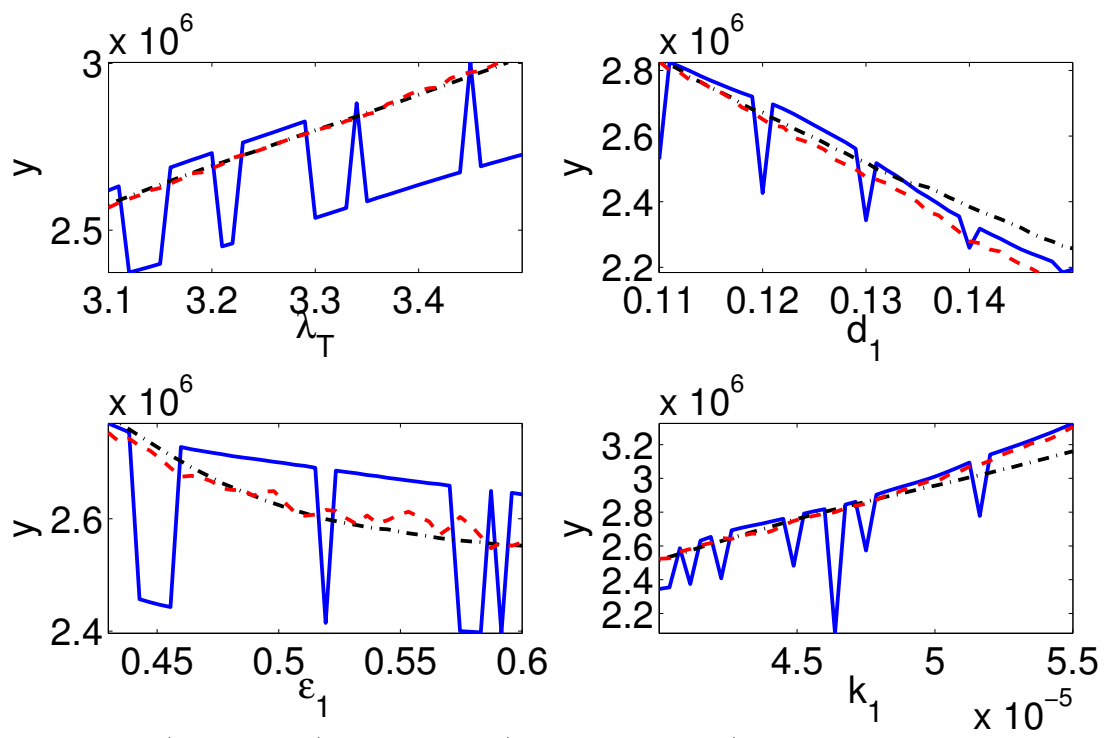

Figure 8: Model response (solid blue), 1st order (dash-dot black) and 2nd order approximation (dash red). 
response, and they do not accurately represent the model response. There are little difference between the first-order and second-order approximations, which explains the similarity between the reported values of $S_{i}$ and $S_{T i}$. In the HIV model (1), the higher order interactions are non-negligible, and the second-order approximation is not sufficiently accurate to completely represent the model response. This may introduce some inaccuracy when determining the relative influence of parameters using the Sobol indices.

\subsection{Morris Screening}

The third method we consider is Morris screening [15, 17. Screening methods rank the importance of parameters by averaging coarse difference relations termed elementary effects. The elementary effects are then used to compute sensitivity measures, based on the mean and variance, which represent the linear effect of parameters and the effect of interaction terms on the model response. Morris Screening employs neighbors to compute elementary effects, which reduces the total model evaluations by approximately a half. Whereas Morris Screening can only rank the parameter importance, and does not quantify the relative importance of each parameter, this method is significantly more efficient than computing Sobol indices. More details regarding the method can be found in [8, 20].

As with Sobol indices, we first map parameters to $[0,1]$. We also assume no prior information about parameters and hence take them to be uniformly distributed. This latter assumption can be modified if prior parameter information is available. The elementary effect is given by

$$
d_{i}(q)=\frac{f\left(q_{1}, \ldots, q_{i-1}, q_{i}+\Delta, q_{i+1}, \ldots, q_{p}\right)-f(q)}{\Delta}=\frac{f\left(q+e_{i}\right)-f(q)}{\Delta},
$$

where $\Delta$ is the step size chosen from the set $\Delta \in\left\{\frac{1}{\ell-1}, \ldots, 1-\frac{1}{\ell-1}\right\}$. Constructed in this way, $d_{i}$ quantifies the approximate, large scale, local sensitivity at $q_{i}$. We note that the step size is taken large to cover the entire parameter space. As detailed in [8, 15, 20, taking $\ell$ to be even and choosing $\Delta=\frac{\ell}{2(\ell-1)}$ has the advantage that it guarantees equal probability sampling from the distribution.

Let

$$
d_{i}^{k}=\frac{f\left(q^{k}+\Delta e_{i}\right)-f\left(q^{k}\right)}{\Delta}
$$

be the elementary effect associated with the $i^{\text {th }}$ parameter and $k^{t h}$ sample. For $r$ sample points, the Morris indices for the parameter $q_{i}$ are

$$
\begin{aligned}
& \mu^{*}=\frac{1}{r} \sum_{k=1}^{r}\left|d_{s}^{k}\right| \\
& \sigma^{2}=\frac{1}{r-1} \sum_{k=1}^{r}\left(d_{s}^{r}-\mu\right)^{2}, \text { where } \mu=\frac{1}{r} \sum_{k=1}^{r} d_{s}^{k} .
\end{aligned}
$$

The mean quantifies the individual effect of the input on output, whereas the variance incorporates the influence of parameter interactions. Since we must consider both the mean and the variance, we rank the parameter using the quantity $\sqrt{\mu^{* 2}+\sigma^{2}}$ when ordering the importance of parameters. Computing (11) requires two model evaluations per parameter per sample. Hence, a total of $2 \mathrm{pr}$ model evaluations is required to compute the Morris indices, $\mu^{*}$ and $\sigma^{2}$. As detailed in Algorithm 3.3.1 below, taken from [8], one employs neighbors to reduce the number of total model evaluations to $(p+1) r$.

\subsubsection{Morris Screening Algorithm}

1. Create a $(p+1) \times p$ matrix $A$ with ones in the lower triangle such that

$$
A=\left[\begin{array}{cccc}
0 & 0 & \ldots & 0 \\
1 & 0 & \ldots & 0 \\
\vdots & & \ddots & \\
1 & 1 & \ldots & 1
\end{array}\right]
$$


2. Choose the step size $\Delta$. Unless specified by the user, take $\Delta=\frac{\ell}{2(\ell-1)}$.

3. Select a starting vector $q^{*}$.

4. Construct a diagonal matrix $D^{*}$, whose entries are randomly chosen from $\{-1,1\}$.

5. Calculate the sampling matrix $A_{s}$ as the following

$$
A_{s}=J_{p+1, p} q^{*}+\frac{\Delta}{2}\left[\left(2 A-J_{p+1, p}\right) D^{*}+J_{p+1, p}\right] P^{*},
$$

where $J_{i, j}$ is a $i \times j$ matrix with all ones and $P^{*}$ is a $p \times p$ permutation of the identity matrix.

6. If the parameters are not defined in the hypercube $[0,1]^{p}$ and instead $q \in\left[\ell b_{i}, u b_{i}\right]$ for $i=1, \ldots, p$, take $\ell b=\left[\ell b_{1}, \ell b_{2}, \ldots \ldots, \ell b_{p}\right]$ and $u b=\left[u b_{1}, u b_{2}, \ldots, u b_{p}\right]$. The sampling matrix is then scaled to match the range of parameters

$$
C=J_{p+1,1} \ell b+A_{s}(D(u b-\ell b))
$$

where $D(u b-\ell b)$ is a diagonal matrix with entries $u b-\ell b$.

7. Compute the elementary effect for $s=1, \ldots, p$. We let $C_{k}$ denote the $k^{t h}$ row of $C$. Then

$$
d_{s}=\frac{f\left(C_{i}\right)-f\left(C_{j}\right)}{\Delta}
$$

where $i$ and $j$ denote the indices such that $i^{\text {th }}$ row and $j^{\text {th }}$ row differ in the $s^{\text {th }}$ entry.

8. Repeat the steps $1-7$ for $r$ samples. The Morris mean $\mu^{*}$ and $\sigma^{2}$ are computed by taking the average of the local elementary effect

$$
\begin{aligned}
& \mu^{*}=\frac{1}{r} \sum_{k=1}^{r}\left|d_{s}^{k}\right| \\
& \sigma^{2}=\frac{1}{r-1} \sum_{k=1}^{r}\left(d_{s}^{r}-\mu\right)^{2}, \text { where } \mu=\frac{1}{r} \sum_{k=1}^{r} d_{s}^{k} .
\end{aligned}
$$

We note that the denominator of (13) in Step 7 is $\Delta$ for all $q_{i}, i=1, \ldots, p$. The elementary effects must be computed using the scaled step size, even though model responses are computed at the parameter values, which are mapped using 12 .

\subsubsection{Morris Indices Results}

We use $\ell=20, r=50$ and the default step size $\Delta=\ell / 2(\ell-1)$. We plot the elementary effects $\mu^{*}$ and $\sigma^{2}$ in Figure 9 to visualize those parameters that are more influential. The most influential parameter is again $N_{T}$ followed by $p_{T}$ and $\epsilon_{2}$. The results also coincide with those from Partial Correlation and Sobol for the least influential parameters, which are $T_{1}^{*}(0)$ and $T_{1}(0)$. The parameter $a_{E}$, which is one of the least influential parameters in Partial Correlation and Sobol after $T_{1}^{*}(0)$ and $T_{1}(0)$, is still ranked low. One difference with Morris screening, however, is that all three initial conditions are identified as minimally influential.

\subsection{Parameter Subset Selection}

Finally, we discuss the parameter subset selection method presented in [3, 4, 6, 9. This method can be used to determine a subset of $n_{p}$ parameters, $n_{p} \leq p$, that are identifiable with the smallest uncertainty measure. The subset selection algorithm uses the optimal parameter estimates as well as standard errors associated with the parameters in the estimation process. We consider a ratio of standard errors and parameter estimates to rank the set of parameters that are most influential for a given $n_{p}$. The parameter subset selection can be used as a parameter selection technique since identifiable parameters are influential, and the $n_{p}$ parameters isolated in this algorithm correspond to the $n_{p}$ most influential parameters. 


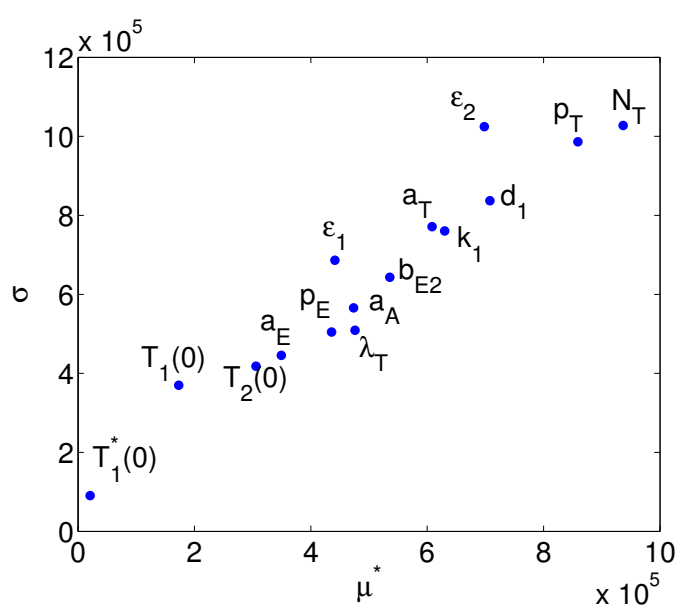

Figure 9: Morris $\mu^{*}$ and $\sigma$ computed using the Morris screening algorithm.

For a vector of parameters $q=\left[q_{1}, \ldots, q_{p}\right]$, we first require the optimal parameter estimates of $q$, denoted by $\hat{q}=\left[\hat{q}_{1}, \ldots, \hat{q}_{p}\right]$, and the corresponding standard errors, $S E=\left[S E_{1}, \ldots, S E_{p}\right]$. Then, given the parameter vector $q$ of size $p$ and a number $n_{p} \leq p$, the subset selection algorithm returns a set of parameters of size $n_{p}$ that minimizes the selection score,

$$
\alpha(\hat{q})=|\nu(\hat{q})| .
$$

Here, $\nu(\hat{q})=\left[\nu\left(\hat{q}_{1}\right), \ldots, \nu\left(\hat{q}_{n_{p}}\right)\right]^{T}$, and $\nu\left(\hat{q}_{i}\right)$ is the coefficient of variation for $\hat{q}_{i}$ defined by

$$
\nu\left(\hat{q}_{i}\right)=\frac{S E_{i}}{\hat{q}_{i}}, \quad i=1, \ldots, n_{p} .
$$

The set of parameters with the smallest selection score gives $n_{p}$ most influential parameters.

\subsubsection{Optimal Parameter Estimates}

This technique utilizes time-dependent responses. Following the strategy in [3], we employ the responses

$$
\begin{aligned}
& z_{1}=T_{1}+T_{1}^{*}+T_{2}+T_{2}^{*} \\
& z_{2}=V_{I}+V_{N I},
\end{aligned}
$$

which are the total CD4+ T-cells and the total RNA copies, respectively. We assume a statistical model of the form

$$
\begin{aligned}
& Y_{1}^{i}=z_{1}\left(t_{1}^{i} ; q_{0}\right)+e_{1}^{i}, i=1,2, \ldots, N_{1} \\
& Y_{2}^{j}=z_{2}\left(t_{2}^{j} ; q_{0}\right)+z_{2}^{\gamma} e_{2}^{j}, j=1,2, \ldots, N_{2},
\end{aligned}
$$

where $y_{1}^{i}$ and $y_{2}^{j}$ are realizations of the random variables $Y_{1}^{i}$ and $Y_{2}^{j}$, respectively, and $e_{1}^{i}$ and $e_{2}^{j}$ are independently identically distributed such that $\mathbb{E}\left[e_{1}^{i}\right]=\mathbb{E}\left[e_{2}^{j}\right]=0$ with $\operatorname{Var}\left(e_{1}^{i}\right)=\sigma_{1}^{2}$ and $\operatorname{Var}\left(e_{2}^{j}\right)=\sigma_{2}^{2}$ for $i=1, \ldots, N_{1}, j=1, \ldots, N_{2}$. Also, $q_{0}$ represents the hypothesized true parameter values.

The weighted least squares estimator is given by

$$
\hat{q}=\underset{q \in Q}{\arg \min }\left(\frac{1}{N_{1}} \sum_{i=1}^{N_{1}} \frac{\left(y_{1}^{i}-z_{1}\left(t_{1}^{i} ; q_{0}\right)\right)^{2}}{\sigma_{1}^{2}}+\frac{1}{N_{2}} \sum_{j=1}^{N_{2}} \frac{\left(y_{2}^{j}-z_{2}\left(t_{2}^{j} ; q_{0}\right)\right)^{2}}{\sigma_{2}^{2} z_{2}^{2 \gamma}\left(t_{2}^{j} ; q_{0}\right)}\right)
$$

where the variance components are given by

$$
\begin{aligned}
& \sigma_{1}^{2}\left(q_{0}\right)=\frac{1}{N_{1}-\operatorname{dim}\left(q_{0}\right)} \sum_{i=1}^{N_{1}}\left(y_{1}^{i}-z_{1}\left(t_{1}^{i} ; q 0\right)\right)^{2} \\
& \sigma_{2}^{2}\left(q_{0}\right)=\frac{1}{N_{2}-\operatorname{dim}\left(q_{0}\right)} \sum_{j=1}^{N_{2}} \frac{\left(y_{2}^{j}-z_{2}\left(t_{2}^{j} ; q 0\right)\right)^{2}}{z_{2}^{2 \gamma}\left(t_{2}^{j} ; q_{0}\right)} .
\end{aligned}
$$


The value of $\gamma$ is determined based on the underlying assumption for the statistical models 15. More specifically, it was determined in [3, 7] that choosing $\gamma=1.2$ results in the residuals being approximately iid, which is an assumption for the model 15 . For this reason, the parameter estimation was performed with $\gamma=1.2$.

Since the estimates in (16) and 17) involve an unknown, to-be-estimated parameter vector $q_{0}$, the optimal parameter is estimated iteratively with the initial variance $\sigma_{k}^{2}=1$ for $k=1,2$ and the weights $z_{2}^{2 \gamma}\left(t_{2}^{j} ; q_{0}\right)=1$ for $j=1, \ldots, N_{2}$. We summarize the parameter estimation algorithm from [3].

\section{Parameter Estimation Procedure Algorithm}

1. Obtain initial estimate $\hat{q}^{(0)}$ using 16 with $\sigma_{k}^{2}=1$ for $k=1,2$ and the weights $z_{2}^{2 \gamma}\left(t_{2}^{j} ; q_{0}\right)=1$ for $j=1, \ldots, N_{2}$.

2. Compute the variances $\sigma_{k}^{2}$ using $(17)$, and the weights $z_{2}^{2 \gamma}\left(t_{2}^{j} ; q_{0}\right)$ with $q_{0}$ replaced by $\hat{q}^{(0)}$.

3. Initialize the iteration counter $\ell$ with the value 1 .

4. Do each of the following:

- Compute $\hat{q}^{(\ell)}$ using 16 with current variances $\sigma_{k}^{2}$ and weights $z_{2}^{2 \gamma}\left(t_{2}^{j} ; \hat{q}^{(\ell-1)}\right)$.

- Update the variances $\sigma_{k}^{2}$ using $(17)$ and the weights $z_{2}^{2 \gamma}\left(t_{2}^{j} ; \hat{q}^{(\ell-1)}\right)$ with $q_{0}, \hat{q}^{(\ell-1)}$ replaced by $\hat{q}^{(\ell)}$.

- Compute $\Delta_{\varepsilon}=\left\|\left[\hat{q}^{(\ell)}-\hat{q}^{(\ell-1)}\right] \cdot /\left[\hat{q}^{(\ell-1)}\right]\right\|$.

- Increment $\ell$ by 1 .

5. If $\Delta_{\varepsilon}>\varepsilon$, go back to Step 4. Otherwise, terminate the algorithm.

In this algorithm, $\varepsilon$ is a user-defined threshold tolerance for a termination criterion, and ./ denotes element-by-element division.

\subsubsection{Computing Standard Errors}

The parameter subset selection algorithm also requires the computation of standard errors for the parameters. The standard errors are computed using standard asymptotic theory for generalized least squares (GLS) estimators $q_{G L S}^{n}$ following the procedure discussed in [3]. The $p \times p$ Fisher Information Matrix (FIM) corresponding to $z_{1}$ and $z_{2}$ in $(14)$ is approximated by

$$
\Sigma_{0}^{N_{1}+N_{2}} \approx\left[\left(\sum_{i=1}^{N_{1}} \frac{1}{\sigma_{1}^{2}\left(\hat{q}^{n}\right)} \frac{\partial z_{1}\left(t_{1}^{i} ; \hat{q}^{n}\right)}{\partial q_{k}} \frac{\partial z_{1}\left(t_{1}^{i} ; \hat{q}^{n}\right)}{\partial q_{\ell}}+\sum_{j=1}^{N_{2}} \frac{1}{\sigma_{2}^{2}\left(\hat{q}^{n}\right) z_{2}^{2 \gamma}\left(t_{2}^{j} ; \hat{q}^{n}\right)} \frac{\partial z_{2}\left(t_{2}^{j} ; \hat{q}^{n}\right)}{\partial q_{k}} \frac{\partial z_{2}\left(t_{2}^{j} ; \hat{q}^{n}\right)}{\partial q_{\ell}}\right)_{k, \ell}\right]
$$

where $\sigma_{1}^{2}$ and $\sigma_{2}^{2}$ are defined in 17 with $q_{0}$ approximated by $\hat{q}^{n}$.

To approximate $q_{0}$, we first let $z_{1}=T_{1}+T_{1}^{*}+T_{2}+T_{2}^{*}$ and $z_{2}=V_{I}+V_{N I}$. The sensitivities are computed by solving the system of equations

$$
\frac{d}{d t}\left(\frac{\partial z_{m}}{\partial q}\right)=\frac{\partial g_{m}}{\partial x}\left(\frac{\partial x}{\partial q}\right)+\frac{d g_{m}}{d q}, m=1,2 .
$$

Here, $x$ and $q$ respectively denote the state variables and the parameters being estimated. Define the $2 \times p$ matrices

$$
\begin{aligned}
& D_{1}^{i}\left(q_{0}\right)=\left[\begin{array}{ccc}
\frac{\partial z_{1}}{\partial q_{1}}\left(t_{1}^{i} ; q_{0}\right) & \ldots & \frac{\partial z_{1}}{\partial q_{p}}\left(t_{1}^{i} ; q_{0}\right) \\
0 & \ldots & 0
\end{array}\right] \text { for } i=1, \ldots, N_{1} \\
& D_{2}^{i}\left(q_{0}\right)=\left[\begin{array}{ccc}
0 & \ldots & 0 \\
\frac{\partial z_{1}}{\partial q_{1}}\left(t_{1}^{i} ; q_{0}\right) & \ldots & \frac{\partial z_{1}}{\partial q_{p}}\left(t_{1}^{i} ; q_{0}\right)
\end{array}\right] \text { for } i=1, \ldots, N_{2} .
\end{aligned}
$$

and define the $2 \times 2$ matrix

$$
V_{0}\left(t ; q_{0}\right)=\left[\begin{array}{cc}
\sigma_{1}^{2} & 0 \\
0 & \sigma_{2}^{2} z_{2}^{2 \gamma}\left(t ; q_{0}\right)
\end{array}\right]
$$


The matrices $D_{1}^{i^{T}} V_{0}^{-1}\left(t_{1}^{i}\right) D_{1}^{i}$ and $D_{2}^{j^{T}} V_{0}^{-1}\left(t_{2}^{j}\right) D_{2}^{j}$ respectively have entries

$$
\begin{aligned}
& F_{k, \ell}^{1, i}\left(q_{0}\right)=\sigma_{1}^{-2} \frac{\partial z_{1}}{\partial q_{k}}\left(t_{1}^{i} ; q_{0}\right) \frac{\partial z_{1}}{\partial q_{\ell}}\left(t_{1}^{i} ; q_{0}\right), k, \ell=1, \ldots, p, i=1, \ldots, N_{1} \\
& F_{k, \ell}^{2, j}\left(q_{0}\right)=\sigma_{2}^{-2} z_{2}^{-2 \gamma}\left(t_{2}^{j} ; q_{0}\right) \frac{\partial z_{2}}{\partial q_{k}}\left(t_{2}^{j} ; q_{0}\right) \frac{\partial z_{2}}{\partial q_{\ell}}\left(t_{2}^{j} ; q_{0}\right), k, \ell=1, \ldots, p, i=1, \ldots, N_{2}
\end{aligned}
$$

Then, we define the $p \times p$ Fisher matrix $F\left(q_{0}\right)=F_{k, \ell}\left(q_{0}\right)$ with entries

$$
F_{k, \ell}\left(q_{0}\right)=\sum_{i=1}^{N_{1}} F_{k, \ell}^{1, i}\left(q_{0}\right)+\sum_{j=1}^{N_{2}} F_{k, \ell}^{2, j}\left(q_{0}\right) .
$$

The approximate Fisher matrix $(18)$ is obtained by evaluating $(19)$ at $\hat{q}^{n} \approx q_{0}$. Using the Fisher matrix approximations, $F$, the standard errors for $\hat{q}_{k}^{n}, k=1, \ldots, p$, are given by

$$
S E_{k}=S E\left(\hat{q}_{k}^{n}\right)=\sqrt{\left(F^{-1}\left(\hat{q}^{n}\right)\right)_{k, k}} \text {. }
$$

It is illustrated in 20] that the standard errors are related to the variance of parameter estimates so they quantify the uncertainty of each parameter. Parameters with small standard errors are estimated with a high degree of certainty, so one can conclude that their impact on the response is influential. On the other hand, parameters that are noninfluential have minimal impact on responses, which yields more uncertainty and larger standard error when estimating optimal parameter values.

\subsubsection{Parameter Subset Selection Results}

As presented in [3], we compile the parameters that give the smallest selection score for a given number of parameters in the set, $n_{p}$, in Table 4. We note that these results are patient-dependent and $N_{T}$ was not in the top three for the considered patient. For other patients, $N_{T}$ is in the top 3.

For example, if we want a subset of three parameters that are most influential, we select $\lambda_{T}, \epsilon_{2}$ and $p_{T}$. In this way, the parameter subset selection algorithm selects a set of parameters for a given number of $n_{p}$; however, it does not specify which parameter is more influential among the selected parameters. We see that the set for $n_{p}=k$ is a subset for $n_{p}=k+1$ for all $k$, except $k=2$. Unlike Partial Correlation, Sobol indices and Morris indices, Parameter Subset Selection has a local sensitivity approach since the sensitivity matrices are computed around the mean values. Nevertheless, we can use the parameter subset selection result to provide a comparison regarding which parameters to include when we specify a number of parameters to choose from the entire set.

\section{Comparison and Verification of Parameter Selection Techniques}

In this section, we illustrate two techniques for verifying the accuracy of the parameter selection techniques. We first verify the results provided by the global sensitivity techniques, which rank the impact or influence of the inputs, and the parameter subset selection. We do this in Section 4.1 by comparing the input rankings provided by the four methods. In Section 4.2, we verify the noninfluential inputs by comparing responses obtained with various input combinations.

\subsection{Verification of Input Rankings}

Here, we provide comparisons of the four methods. First, we summarize in Table 5 sensitivity measures, a description and the computational cost of each method. The Sobol indices, Morris indices and Partial Correlation indices are summarized in Table 6 in order of importance. For Partial Correlation, we rank the importance by the absolute values of the partial correlation. For the Sobol indices, the parameters are ranked by the magnitude of $S_{T i}$. For the Morris indices, we consider the quantity $\sqrt{\mu^{* 2}+\sigma^{2}}$ to rank the parameters. 


\begin{tabular}{|c|c|c|c|c|c|c|c|c|c|c|c|c|c|c|c|}
\hline$n_{p}$ & $N_{T}$ & $\lambda_{T}$ & $\epsilon_{2}$ & $p_{T}$ & $p_{E}$ & $T_{2}(0)$ & $T_{1}(0)$ & $\epsilon_{1}$ & $d_{1}$ & $b_{E 2}$ & $a_{E}$ & $a_{T}$ & $k_{1}$ & $a_{A}$ & $T_{1}^{*}(0)$ \\
\hline 1 & $\mathrm{x}$ & & & & & & & & & & & & & & \\
\hline 2 & $\mathrm{x}$ & $\mathrm{x}$ & & & & & & & & & & & & & \\
\hline 3 & & $\mathrm{X}$ & $\mathrm{x}$ & $\mathrm{x}$ & & & & & & & & & & & \\
\hline 4 & $\mathrm{x}$ & $\mathrm{x}$ & $\mathrm{x}$ & $\mathrm{x}$ & & & & & & & & & & & \\
\hline 5 & $\mathrm{x}$ & $\mathrm{x}$ & $\mathrm{X}$ & $\mathrm{X}$ & $\mathrm{x}$ & & & & & & & & & & \\
\hline 6 & $\mathrm{x}$ & $\mathrm{X}$ & $\mathrm{x}$ & $\mathrm{X}$ & $\mathrm{X}$ & $x$ & & & & & & & & & \\
\hline 7 & $x$ & $\mathrm{x}$ & $\mathrm{x}$ & $\mathrm{x}$ & $\mathrm{x}$ & $\mathrm{x}$ & $\mathrm{x}$ & & & & & & & & \\
\hline 8 & $\mathrm{x}$ & $\mathrm{x}$ & $\mathrm{x}$ & $\mathrm{x}$ & $\mathrm{X}$ & $\mathrm{x}$ & $\mathrm{x}$ & $\mathrm{x}$ & & & & & & & \\
\hline 9 & $\mathrm{x}$ & $\mathrm{x}$ & $\mathrm{x}$ & $\mathrm{X}$ & $\mathrm{X}$ & $\mathrm{x}$ & $\mathrm{X}$ & $\mathrm{X}$ & $\mathrm{X}$ & & & & & & \\
\hline 10 & $\mathrm{x}$ & $\mathrm{X}$ & $\mathrm{x}$ & $\mathrm{X}$ & $\mathrm{x}$ & $\mathrm{x}$ & $\mathrm{x}$ & $\mathrm{x}$ & $\mathrm{x}$ & $\mathrm{X}$ & & & & & \\
\hline 11 & $\mathrm{x}$ & $\mathrm{x}$ & $\mathrm{x}$ & $\mathrm{x}$ & $\mathrm{X}$ & $\mathrm{x}$ & $\mathrm{x}$ & $\mathrm{x}$ & $\mathrm{x}$ & $\mathrm{x}$ & $\mathrm{x}$ & & & & \\
\hline 12 & $\mathrm{x}$ & $\mathrm{x}$ & $\mathrm{x}$ & $\mathrm{x}$ & $\mathrm{x}$ & $\mathrm{x}$ & $\mathrm{x}$ & $\mathrm{x}$ & $\mathrm{X}$ & $\mathrm{X}$ & $\mathrm{x}$ & $\mathrm{X}$ & & & \\
\hline 13 & $\mathrm{x}$ & $\mathrm{x}$ & $\mathrm{x}$ & $\mathrm{x}$ & $\mathrm{x}$ & $\mathrm{X}$ & $\mathrm{X}$ & $\mathrm{x}$ & $\mathrm{X}$ & $x$ & $x$ & $\mathrm{x}$ & $\mathrm{x}$ & & \\
\hline 14 & $\mathrm{x}$ & $\mathrm{x}$ & $\mathrm{x}$ & $\mathrm{x}$ & $\mathrm{x}$ & $\mathrm{x}$ & $\mathrm{x}$ & $\mathrm{x}$ & $\mathrm{X}$ & $\mathrm{x}$ & $\mathrm{x}$ & $\mathrm{x}$ & $\mathrm{x}$ & $\mathrm{x}$ & \\
\hline 15 & $\mathrm{x}$ & $\mathrm{x}$ & $x$ & $\mathrm{x}$ & $\mathrm{x}$ & $\mathrm{x}$ & $\mathrm{x}$ & $\mathrm{x}$ & $\mathrm{x}$ & $\mathrm{x}$ & $\mathrm{x}$ & $\mathrm{x}$ & $\mathrm{x}$ & $\mathrm{x}$ & $\mathrm{x}$ \\
\hline
\end{tabular}

Table 4: Parameter subset selection results from [3].

To provide a comparison among the four methods, we summarize in Table 7 and 8 the parameters to be selected for a given number of parameters. In Table 7 and 8. PCorr, S, M and PSS respectively denote Partial Correlation, Sobol indices via Saltelli algorithm, Morris indices and Parameter Subset Selection. For Partial Correlation, Sobol indices and Morris indices, $n_{p}$ influential parameters correspond to the top $n_{p}$ parameters from Table 6

Overall, Partial Correlation is the cheapest method to measure linearity between parameters and response. This often corresponds to the first order Sobol indices. Computing Sobol indices is expensive and it becomes prohibitively slow as the number of input parameters increases. For a model with a moderate number of input parameters, we can apply Morris screening. This employs neighbors to compute statistically averaged local, very coarse approximations to derivatives. Morris indices are a good measure to isolate influential parameters from noninfluential parameters with much fewer evaluations than Sobol indices. Finally, the parameter subset selection algorithm provides sensitivity in terms of uncertainties involved in the estimation process. The noninfluential parameters determined by this method did not match the results from the other three.

In terms of accuracy, Sobol indices measure the first- and second-order interaction effects of parameters most accurately. However, we showed that second-order Sobol decomposition may not be sufficiently accurate depending on the model. Even though the Sobol indices are widely used for global sensitivity analysis, one must always consider the accuracy of Sobol decomposition as an approximation to the model before applying the results of Sobol indices.

When the parameter selection techniques are applied to the HIV model, we found that certain parameters are determined highly influential by all four methods. An example of highly influential parameters are $N_{T}$ and $p_{T}$. These parameters respectively represent the number of RNA copies during the process of $T_{1}^{*}$ lysis and net proliferation of $T_{1}$ and $T_{1}$ due to clonal expansion and programmed contraction. We also observed that both $\epsilon_{1}$ and $\epsilon_{2}$ were ranked above average in their importance. This is essential in designing the optimal control for drug therapy. We see from our global sensitivity analysis that the relative effectiveness of protease inhibitor, $\epsilon_{2}$, has more affect on the model response than that of reverse transcriptase inhibitor, $\epsilon_{1}$. On the other hand, for our specific response, it was shown that initial conditions $T_{1}(0), T_{1}^{*}(0)$ and $T_{2}(0)$ do not play a strong role in determining the response.

\subsection{Verification of Noninfluential Inputs}

To verify that the influential parameters are correctly identified, we compute the probability density functions of model responses by fixing noninfluential parameters. 


\begin{tabular}{|l|l|l|l|}
\hline Method & Sensitivity Measure & Description & Cost \\
\hline \hline $\begin{array}{l}\text { Partial } \\
\text { Correlation }\end{array}$ & $\begin{array}{l}\text { Degree of linear corre- } \\
\text { lation between param- } \\
\text { eters and response }\end{array}$ & $\begin{array}{l}\text { Ranks the parameters in the order of } \\
\text { strong linear correlation to response. } \\
\text { Considers linearity only. }\end{array}$ & $\begin{array}{l}M \text { model evaluations } \\
\text { for } M \text { Monte Carlo } \\
\text { samples. }\end{array}$ \\
\hline $\begin{array}{l}\text { Sobol by } \\
\text { Saltelli }\end{array}$ & $\begin{array}{l}\text { First order Sobol } S_{i} \\
\text { and total sensitivity in- } \\
\text { dices } S_{T i}\end{array}$ & $\begin{array}{l}\text { A type of variance-based method. Uses } \\
\text { 2nd order Sobol decomposition. Ranks } \\
\text { the parameters and quantifies relative } \\
\text { importance. Measures the effects of in- } \\
\text { dividual parameters as well as interac- } \\
\text { tion terms. }\end{array}$ & $\begin{array}{l}\text { ations for } M \text { Monte } \\
\text { Carlo samples and } p \\
\text { parameters. }\end{array}$ \\
\hline $\begin{array}{l}\text { Morris } \\
\text { Screening }\end{array}$ & $\begin{array}{l}\text { Mean } \mu_{i}^{*} \text { and variance } \\
\sigma^{2} \text { of elementary ef- } \\
\text { fects }\end{array}$ & $\begin{array}{l}\text { Averages coarse local derivative ap- } \\
\text { proximations. Only ranks parameters. } \\
\text { Employs neighbors to reduce the cost. }\end{array}$ & $\begin{array}{l}(p+1) r \text { model eval- } \\
\text { uations for } r \text { sample } \\
\text { points for averaging } \\
\text { and } p \text { parameters. }\end{array}$ \\
\hline $\begin{array}{l}\text { Parameter } \\
\text { Subset } \\
\text { Selection }\end{array}$ & $\begin{array}{l}n_{p} \text { parameters with } \\
\text { minimum uncertainty }\end{array}$ & $\begin{array}{l}\text { Provides identifiable subset of } n_{p} \text { pa- } \\
\text { rameters. Requires the optimal param- } \\
\text { eter estimate } \hat{q} \text { and standard errors } S E .\end{array}$ & $\begin{array}{l}C\left(p, n_{p}\right) \text { subsets to } \\
\text { check for minimum } \\
\text { uncertainty for sub- } \\
\text { set of } n_{p} \text { parameters } \\
\text { among } p \text { parameters. }\end{array}$ \\
\hline
\end{tabular}

Table 5: Summary of parameter selection techniques.

\begin{tabular}{|c||cr||rrr||cr|}
\hline \multicolumn{1}{|c||}{} & \multicolumn{1}{c||}{ Partial Correlation } & \multicolumn{3}{c||}{ Sobol by Saltelli } & \multicolumn{2}{c|}{ Morris Indices } \\
\hline Rank & Parameter & Corr $(q, y)$ & Parameter & $S_{i}$ & $S_{T i}$ & Parameter & $\sqrt{\mu^{* 2}+\sigma^{2}}$ \\
\hline 1 & $N_{T}$ & $4.608 \mathrm{e}-1$ & $N_{T}$ & $1.455 \mathrm{e}-1$ & $2.134 \mathrm{e}-1$ & $N_{T}$ & $1.390 \mathrm{e}+6$ \\
2 & $p_{T}$ & $3.964 \mathrm{e}-1$ & $p_{T}$ & $1.046 \mathrm{e}-1$ & $2.038 \mathrm{e}-1$ & $p_{T}$ & $1.308 \mathrm{e}+6$ \\
3 & $k_{1}$ & $2.970 \mathrm{e}-1$ & $k_{1}$ & $1.066 \mathrm{e}-1$ & $1.335 \mathrm{e}-1$ & $\epsilon_{2}$ & $1.240 \mathrm{e}+6$ \\
4 & $d_{1}$ & $-2.630 \mathrm{e}-1$ & $\epsilon_{2}$ & $7.879 \mathrm{e}-3$ & $1.329 \mathrm{e}-1$ & $d_{1}$ & $1.096 \mathrm{e}+6$ \\
5 & $b_{E 2}$ & $-2.526 \mathrm{e}-1$ & $d_{1}$ & $6.276 \mathrm{e}-2$ & $1.141 \mathrm{e}-1$ & $k_{1}$ & $9.876 \mathrm{e}+5$ \\
6 & $a_{T}$ & $2.178 \mathrm{e}-1$ & $a_{T}$ & $5.568 \mathrm{e}-2$ & $9.541 \mathrm{e}-2$ & $a_{T}$ & $9.824 \mathrm{e}+5$ \\
7 & $\epsilon_{2}$ & $-2.167 \mathrm{e}-1$ & $\epsilon_{1}$ & $5.426 \mathrm{e}-2$ & $7.849 \mathrm{e}-2$ & $b_{E 2}$ & $8.371 \mathrm{e}+5$ \\
8 & $a_{A}$ & $2.111 \mathrm{e}-1$ & $b_{E 2}$ & $8.948 \mathrm{e}-2$ & $7.723 \mathrm{e}-2$ & $\epsilon_{1}$ & $8.161 \mathrm{e}+5$ \\
9 & $p_{E}$ & $-1.789 \mathrm{e}-1$ & $a_{A}$ & $3.727 \mathrm{e}-2$ & $5.458 \mathrm{e}-2$ & $a_{A}$ & $7.378 \mathrm{e}+5$ \\
10 & $\lambda_{T}$ & $1.774 \mathrm{e}-1$ & $p_{E}$ & $2.013 \mathrm{e}-2$ & $5.384 \mathrm{e}-2$ & $\lambda_{T}$ & $6.971 \mathrm{e}+5$ \\
11 & $\epsilon_{1}$ & $-1.601 \mathrm{e}-1$ & $\lambda_{T}$ & $4.682 \mathrm{e}-2$ & $4.626 \mathrm{e}-2$ & $p_{E}$ & $6.666 \mathrm{e}+5$ \\
12 & $T_{2}(0)$ & $1.487 \mathrm{e}-1$ & $T_{2}(0)$ & $-1.032 \mathrm{e}-2$ & $4.266 \mathrm{e}-2$ & $a_{E}$ & $5.662 \mathrm{e}+5$ \\
13 & $a_{E}$ & $-9.089 \mathrm{e}-2$ & $a_{E}$ & $1.057 \mathrm{e}-2$ & $1.943 \mathrm{e}-2$ & $T_{2}(0)$ & $5.180 \mathrm{e}+5$ \\
14 & $T_{1}^{*}(0)$ & $-2.721 \mathrm{e}-2$ & $T_{1}(0)$ & $-3.974 \mathrm{e}-3$ & $1.464 \mathrm{e}-2$ & $T_{1}(0)$ & $4.084 \mathrm{e}+5$ \\
15 & $T_{1}(0)$ & $2.410 \mathrm{e}-2$ & $T_{1}^{*}(0)$ & $-8.715 \mathrm{e}-3$ & $2.423 \mathrm{e}-3$ & $T_{1}^{*}(0)$ & $9.285 \mathrm{e}+4$ \\
\hline
\end{tabular}

Table 6: Sensitivity measures provided by Partial Correlation, Sobol by the Saltelli algorithm and Morris Screening. 


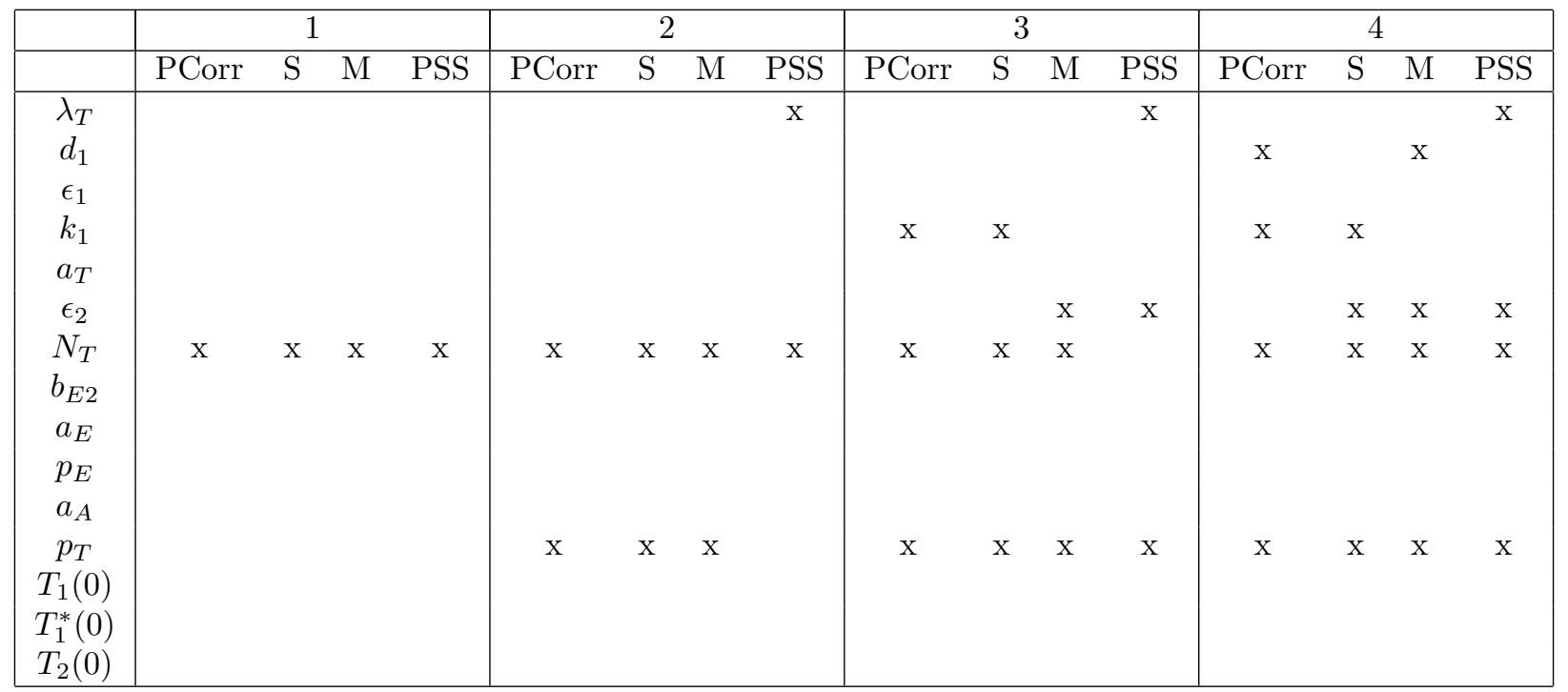

\begin{tabular}{|c|c|c|c|c|c|c|c|c|c|c|c|c|c|c|c|c|}
\hline & \multicolumn{4}{|c|}{5} & \multicolumn{4}{|c|}{6} & \multicolumn{4}{|c|}{7} & \multicolumn{4}{|c|}{8} \\
\hline & PCorr & $\mathrm{S}$ & $\mathrm{M}$ & PSS & PCorr & $\mathrm{S}$ & M & PSS & PCorr & $\mathrm{S}$ & $\mathrm{M}$ & PSS & PCorr & $\mathrm{S}$ & $\mathrm{M}$ & PSS \\
\hline$\lambda_{T}$ & & & & $\mathrm{x}$ & & & & $\mathrm{x}$ & & & & $\mathrm{x}$ & & & & $\mathrm{x}$ \\
\hline$d_{1}$ & $\mathrm{x}$ & $\mathrm{x}$ & $\mathrm{x}$ & & $\mathrm{x}$ & $\mathrm{x}$ & $\mathrm{x}$ & & $\mathrm{x}$ & $\mathrm{x}$ & $\mathrm{x}$ & & $\mathrm{x}$ & $\mathrm{x}$ & $\mathrm{x}$ & \\
\hline$\epsilon_{1}$ & & & & & & & & & & $\mathrm{x}$ & & & & $\mathrm{x}$ & $\mathrm{x}$ & $\mathrm{x}$ \\
\hline$k_{1}$ & $\mathrm{x}$ & $\mathrm{x}$ & $\mathrm{x}$ & & $\mathrm{x}$ & $\mathrm{x}$ & $\mathrm{x}$ & & $\mathrm{x}$ & $\mathrm{x}$ & $\mathrm{x}$ & & $\mathrm{x}$ & $\mathrm{x}$ & $\mathrm{x}$ & \\
\hline$a_{T}$ & & & & & $\mathrm{x}$ & $\mathrm{x}$ & $\mathrm{x}$ & & $\mathrm{x}$ & $\mathrm{x}$ & $\mathrm{x}$ & & $\mathrm{x}$ & $\mathrm{x}$ & $\mathrm{x}$ & \\
\hline$\epsilon_{2}$ & & $\mathrm{x}$ & $\mathrm{x}$ & $\mathrm{x}$ & & $\mathrm{x}$ & $\mathrm{x}$ & $\mathrm{x}$ & $\mathrm{x}$ & $\mathrm{x}$ & $\mathrm{x}$ & $\mathrm{x}$ & $\mathrm{x}$ & $\mathrm{x}$ & $\mathrm{x}$ & $\mathrm{x}$ \\
\hline$N_{T}$ & $\mathrm{x}$ & $\mathrm{x}$ & $\mathrm{x}$ & $\mathrm{x}$ & $\mathrm{x}$ & $\mathrm{x}$ & $\mathrm{x}$ & $\mathrm{x}$ & $\mathrm{x}$ & $\mathrm{x}$ & $\mathrm{x}$ & $\mathrm{x}$ & $\mathrm{x}$ & $\mathrm{x}$ & $\mathrm{x}$ & $\mathrm{x}$ \\
\hline $\begin{array}{c}b_{E 2} \\
a_{E}\end{array}$ & $\mathrm{x}$ & & & & $\mathrm{x}$ & & & & $\mathrm{x}$ & & $\mathrm{x}$ & & $\mathrm{x}$ & $\mathrm{x}$ & $\mathrm{x}$ & \\
\hline$p_{E}$ & & & & $\mathrm{x}$ & & & & $\mathrm{x}$ & & & & $\mathrm{x}$ & & & & $\mathrm{x}$ \\
\hline$a_{A}$ & & & & & & & & & & & & & $\mathrm{x}$ & & & \\
\hline$p_{T}$ & $\mathrm{x}$ & $\mathrm{x}$ & $\mathrm{x}$ & $\mathrm{x}$ & $\mathrm{x}$ & $\mathrm{x}$ & $\mathrm{x}$ & $\mathrm{x}$ & $\mathrm{x}$ & $\mathrm{x}$ & $\mathrm{x}$ & $\mathrm{x}$ & $\mathrm{x}$ & $\mathrm{x}$ & $\mathrm{x}$ & $\mathrm{x}$ \\
\hline$T_{1}(0)$ & & & & & & & & & & & & $\mathrm{x}$ & & & & $\mathrm{x}$ \\
\hline$T_{1}^{*}(0)$ & & & & & & & & & & & & & & & & \\
\hline$T_{2}(0)$ & & & & & & & & $\mathrm{x}$ & & & & $\mathrm{x}$ & & & & $\mathrm{x}$ \\
\hline
\end{tabular}

Table 7: Subsets of influential parameters for $n_{p}=1, \ldots, 8$. Here, PCorr, S, M and PSS denote Partial Correlations, Sobol indices, Morris indices and Parameter Subset Selection. 


\begin{tabular}{|c|c|c|c|c|c|c|c|c|c|c|c|c|c|c|c|c|}
\hline & \multicolumn{4}{|c|}{9} & \multicolumn{4}{|c|}{10} & \multicolumn{4}{|c|}{11} & \multicolumn{4}{|c|}{12} \\
\hline & PCorr & $\mathrm{S}$ & M & PSS & PCorr & $\mathrm{S}$ & $\mathrm{M}$ & PSS & PCorr & $\mathrm{S}$ & $\mathrm{M}$ & PSS & PCorr & $\mathrm{S}$ & $\bar{M}$ & PSS \\
\hline$\lambda_{T}$ & & & & $\mathrm{x}$ & $\mathrm{x}$ & & $\mathrm{x}$ & $\mathrm{x}$ & $\mathrm{x}$ & $\mathrm{x}$ & $\mathrm{x}$ & $\mathrm{x}$ & $\mathrm{x}$ & $\mathrm{x}$ & $\mathrm{x}$ & $\mathrm{x}$ \\
\hline$d_{1}$ & $\mathrm{x}$ & $\mathrm{x}$ & $\mathrm{x}$ & $\mathrm{x}$ & $\mathrm{x}$ & $\mathrm{x}$ & $\mathrm{x}$ & $\mathrm{x}$ & $\mathrm{x}$ & $\mathrm{x}$ & $\mathrm{x}$ & $\mathrm{x}$ & $\mathrm{x}$ & $\mathrm{x}$ & $\mathrm{x}$ & $\mathrm{x}$ \\
\hline$\epsilon_{1}$ & & $\mathrm{x}$ & $\mathrm{x}$ & $\mathrm{x}$ & & $\mathrm{x}$ & $\mathrm{x}$ & $\mathrm{x}$ & $\mathrm{x}$ & $\mathrm{x}$ & $\mathrm{x}$ & $\mathrm{x}$ & $\mathrm{x}$ & $\mathrm{x}$ & $\mathrm{x}$ & $\mathrm{x}$ \\
\hline$k_{1}$ & $\mathrm{x}$ & $\mathrm{x}$ & $\mathrm{x}$ & & $\mathrm{x}$ & $\mathrm{x}$ & $\mathrm{x}$ & & $\mathrm{x}$ & $\mathrm{x}$ & $\mathrm{x}$ & & $\mathrm{x}$ & $\mathrm{x}$ & $\mathrm{x}$ & \\
\hline$a_{T}$ & $\mathrm{x}$ & $\mathrm{x}$ & $\mathrm{x}$ & & $\mathrm{x}$ & $\mathrm{x}$ & $\mathrm{x}$ & & $\mathrm{x}$ & $\mathrm{x}$ & $\mathrm{x}$ & & $\mathrm{x}$ & $\mathrm{x}$ & $\mathrm{x}$ & $\mathrm{x}$ \\
\hline$\epsilon_{2}$ & $\mathrm{x}$ & $\mathrm{x}$ & $\mathrm{x}$ & $\mathrm{x}$ & $\mathrm{x}$ & $\mathrm{x}$ & $\mathrm{x}$ & $\mathrm{x}$ & $\mathrm{x}$ & $\mathrm{x}$ & $\mathrm{x}$ & $\mathrm{x}$ & $\mathrm{x}$ & $\mathrm{x}$ & $\mathrm{x}$ & $\mathrm{x}$ \\
\hline$N_{T}$ & $\mathrm{x}$ & $\mathrm{x}$ & $\mathrm{x}$ & $\mathrm{x}$ & $\mathrm{x}$ & $\mathrm{x}$ & $\mathrm{x}$ & $\mathrm{x}$ & $\mathrm{x}$ & $\mathrm{x}$ & $\mathrm{x}$ & $\mathrm{x}$ & $\mathrm{x}$ & $\mathrm{x}$ & $\mathrm{x}$ & $\mathrm{x}$ \\
\hline$b_{E 2}$ & $\mathrm{x}$ & $\mathrm{x}$ & $\mathrm{x}$ & & $\mathrm{x}$ & $\mathrm{x}$ & $\mathrm{x}$ & $\mathrm{x}$ & $\mathrm{x}$ & $\mathrm{x}$ & $\mathrm{x}$ & $\mathrm{x}$ & $\mathrm{x}$ & $\mathrm{x}$ & $\mathrm{x}$ & $\mathrm{x}$ \\
\hline$a_{E}$ & & & & & & & & & & & & $\mathrm{X}$ & & & $\mathrm{x}$ & $\mathrm{x}$ \\
\hline$p_{E}$ & $\mathrm{x}$ & & & $\mathrm{x}$ & $\mathrm{x}$ & $\mathrm{x}$ & & $\mathrm{x}$ & $\mathrm{x}$ & $\mathrm{x}$ & $\mathrm{x}$ & $\mathrm{x}$ & $\mathrm{x}$ & $\mathrm{x}$ & $\mathrm{x}$ & $\mathrm{x}$ \\
\hline$a_{A}$ & $\mathrm{x}$ & $\mathrm{x}$ & $\mathrm{x}$ & & $\mathrm{x}$ & $\mathrm{x}$ & $\mathrm{x}$ & & $\mathrm{x}$ & $\mathrm{x}$ & $\mathrm{x}$ & & $\mathrm{x}$ & $\mathrm{x}$ & $\mathrm{x}$ & \\
\hline$p_{T}$ & $\mathrm{x}$ & $\mathrm{x}$ & $\mathrm{x}$ & $\mathrm{x}$ & $\mathrm{x}$ & $\mathrm{x}$ & $\mathrm{x}$ & $\mathrm{x}$ & $\mathrm{x}$ & $\mathrm{x}$ & $\mathrm{x}$ & $\mathrm{x}$ & $\mathrm{x}$ & $\mathrm{x}$ & $\mathrm{x}$ & $\mathrm{x}$ \\
\hline$T_{1}(0)$ & & & & $\mathrm{x}$ & & & & $\mathrm{x}$ & & & & $\mathrm{x}$ & & & & $\mathrm{x}$ \\
\hline$T_{1}^{*}(0)$ & & & & & & & & & & & & & & & & \\
\hline$T_{2}(0)$ & & & & $\mathrm{x}$ & & & & $\mathrm{x}$ & & & & $\mathrm{x}$ & $\mathrm{x}$ & $\mathrm{x}$ & & $\mathrm{x}$ \\
\hline
\end{tabular}

\begin{tabular}{|c|cccc|cccc|}
\hline & \multicolumn{5}{|c|}{13} & \multicolumn{4}{|c|}{14} \\
\hline & PCorr & $\mathrm{S}$ & $\mathrm{M}$ & $\mathrm{PSS}$ & $\mathrm{PCorr}$ & $\mathrm{S}$ & $\mathrm{M}$ & $\mathrm{PSS}$ \\
\hline$\lambda_{T}$ & $\mathrm{x}$ & $\mathrm{x}$ & $\mathrm{x}$ & $\mathrm{x}$ & $\mathrm{x}$ & $\mathrm{x}$ & $\mathrm{x}$ & $\mathrm{x}$ \\
$d_{1}$ & $\mathrm{x}$ & $\mathrm{x}$ & $\mathrm{x}$ & $\mathrm{x}$ & $\mathrm{x}$ & $\mathrm{x}$ & $\mathrm{x}$ & $\mathrm{x}$ \\
$\epsilon_{1}$ & $\mathrm{x}$ & $\mathrm{x}$ & $\mathrm{x}$ & $\mathrm{x}$ & $\mathrm{x}$ & $\mathrm{x}$ & $\mathrm{x}$ & $\mathrm{x}$ \\
$k_{1}$ & $\mathrm{x}$ & $\mathrm{x}$ & $\mathrm{x}$ & $\mathrm{x}$ & $\mathrm{x}$ & $\mathrm{x}$ & $\mathrm{x}$ & $\mathrm{x}$ \\
$a_{T}$ & $\mathrm{x}$ & $\mathrm{x}$ & $\mathrm{x}$ & $\mathrm{x}$ & $\mathrm{x}$ & $\mathrm{x}$ & $\mathrm{x}$ & $\mathrm{x}$ \\
$\epsilon_{2}$ & $\mathrm{x}$ & $\mathrm{x}$ & $\mathrm{x}$ & $\mathrm{x}$ & $\mathrm{x}$ & $\mathrm{x}$ & $\mathrm{x}$ & $\mathrm{x}$ \\
$N_{T}$ & $\mathrm{x}$ & $\mathrm{x}$ & $\mathrm{x}$ & $\mathrm{x}$ & $\mathrm{x}$ & $\mathrm{x}$ & $\mathrm{x}$ & $\mathrm{x}$ \\
$b_{E 2}$ & $\mathrm{x}$ & $\mathrm{x}$ & $\mathrm{x}$ & $\mathrm{x}$ & $\mathrm{x}$ & $\mathrm{x}$ & $\mathrm{x}$ & $\mathrm{x}$ \\
$a_{E}$ & $\mathrm{x}$ & $\mathrm{x}$ & $\mathrm{x}$ & $\mathrm{x}$ & $\mathrm{x}$ & $\mathrm{x}$ & $\mathrm{x}$ & $\mathrm{x}$ \\
$p_{E}$ & $\mathrm{x}$ & $\mathrm{x}$ & $\mathrm{x}$ & $\mathrm{x}$ & $\mathrm{x}$ & $\mathrm{x}$ & $\mathrm{x}$ & $\mathrm{x}$ \\
$a_{A}$ & $\mathrm{x}$ & $\mathrm{x}$ & $\mathrm{x}$ & & $\mathrm{x}$ & $\mathrm{x}$ & $\mathrm{x}$ & $\mathrm{x}$ \\
$p_{T}$ & $\mathrm{x}$ & $\mathrm{x}$ & $\mathrm{x}$ & $\mathrm{x}$ & $\mathrm{x}$ & $\mathrm{x}$ & $\mathrm{x}$ & $\mathrm{x}$ \\
$T_{1}(0)$ & & & & $\mathrm{x}$ & & $\mathrm{x}$ & $\mathrm{x}$ & $\mathrm{x}$ \\
$T_{1}^{*}(0)$ & & & & & $\mathrm{x}$ & & & \\
$T_{2}(0)$ & $\mathrm{x}$ & $\mathrm{x}$ & $\mathrm{x}$ & $\mathrm{x}$ & $\mathrm{x}$ & $\mathrm{x}$ & $\mathrm{x}$ & $\mathrm{x}$ \\
\hline
\end{tabular}

Table 8: Subsets of influential parameters for $n_{p}=9, \ldots, 14$. 


\section{Verification Procedure}

1. For a set of $n_{p}$ influential parameters, sample $n=1000$ parameter values from their respective distributions. For the results reported here, we took the distributions to be uniform with lower and upper bounds summarized in Table 3 .

2. Fix $p-n_{p}$ noninfluential parameters at pre-specified values, which we take to be the lower bounds of the parameters.

3. Compute the model response with parameter values from Steps 1 and 2.

4. Construct probability density function using a kernel density estimation.

We then compare the densities for the model responses where all parameters are sampled randomly. We construct densities for $n_{p}=8,10,12,14$ influential parameters. That is, we examine four cases where the numbers of fixed parameters are 7, 5, 3 and 1, and plot the densities along with the density obtained by varying all the parameters. In Figure 10 (a) and (b), we see that we have fixed too many parameters. In Figure 10 (c), densities using Partial Correlation, Sobol and Morris match the sample density, whereas the density from parameter subset selection does not match the rest. This is reasonable since the influential parameters determined via Partial Correlation, Sobol and Morris indices are very similar as shown in Table 7. Finally in Figure 10 (d), we see that all four methods give comparable densities. The agreement of densities indicates that the parameter $T^{*}(0)$ was determined to be noninfluential and it did not affect the output significantly. Moreover, this is the only parameter that can be fixed without affecting this output.

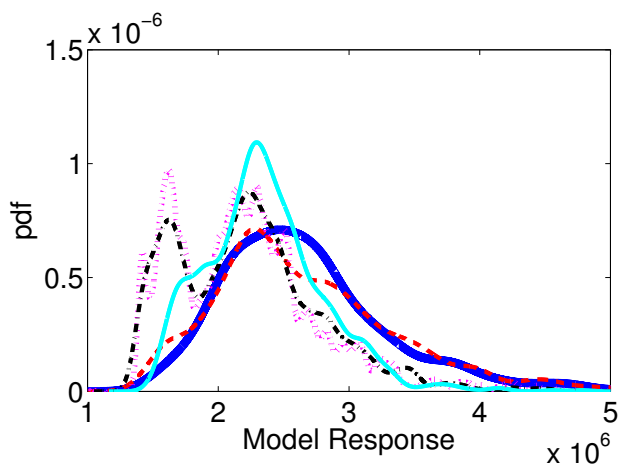

(a)

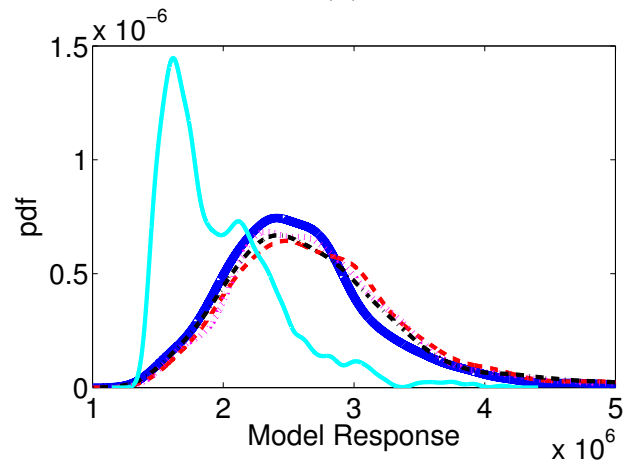

(c)

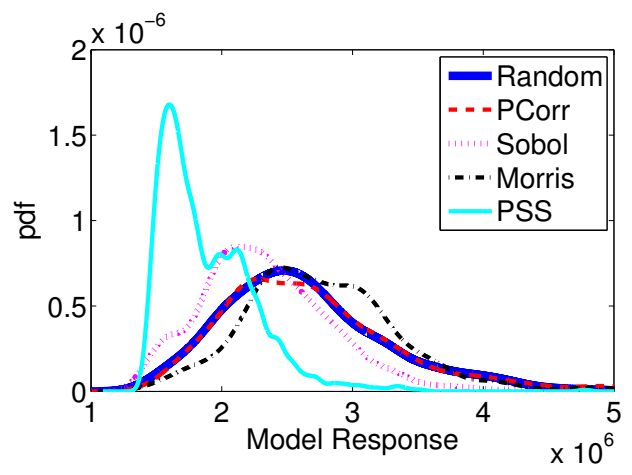

(b)

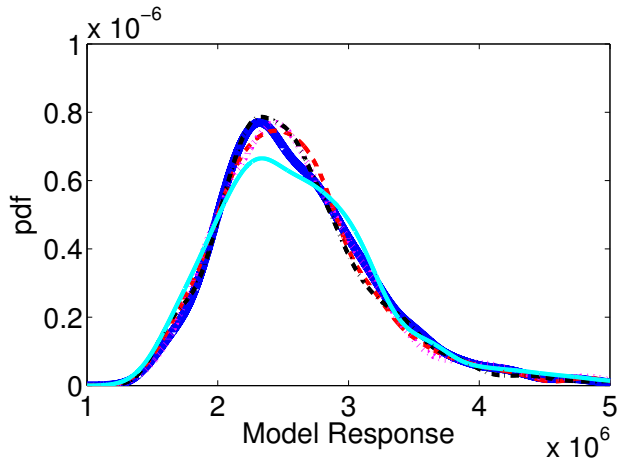

(d)

Figure 10: Densities obtained by fixing (a) 7, (b) 5, (c) 3 and (d) 1 least influential parameters. The density in solid is obtained by varying all the parameters.

In terms of parameter estimation to be followed after parameter selection, there are two inherent difficulties. First, the cut-off between influential and noninfluential parameters is not always clear. Depending on the model, one might observe a cluster of parameters with high sensitivity measures and another cluster with lower sensitivity measures. For the HIV example, it was only after we performed a verification test that we 


\begin{tabular}{|c||c|cc|cc|}
\hline Parameter & Partial Correlation & $S$ & $S_{T}$ & $\mu^{*}$ & $\sigma^{2}$ \\
\hline$q_{1}$ & 0.690 & 0.498 & 0.516 & 1 & 0.980 \\
$q_{2}$ & -0.700 & 0.481 & 0.498 & 1 & 1.020 \\
\hline
\end{tabular}

Table 9: Sensitivity measures for $y=q_{1}-q_{2}$.

learned that fixing one parameter resulted in insignificant variability of sample densities. This verification requires additional model evaluations and there is not a simple way to check which parameters are influential just by observing sensitivity measures.

Second, even if we are successful at isolating influential parameters, the parameter identifiability issues may still remain. Consider a simple example

$$
y=q_{1}-q_{2}
$$

with $q_{1}, q_{2} \sim U(0,1)$. As we can see from the sensitivity measures summarized in Table $9 q_{1}$ and $q_{2}$ are equally influential. Suppose that we have the observation $y=0$. It is easy to see that parameter estimation using this observation will fail to estimate the densities of $q_{1}$ and $q_{2}$ correctly since there are several values of $q_{1}$ and $q_{2}$ that match the observation. Therefore, unless some prior knowledge is specified, $q_{1}$ and $q_{2}$ are unidentifiable.

This simple example illustrates that determining influential parameters may not eliminate parameter identifiability issues completely. In this regard, the parameter subset selection algorithm has the advantage that the selected subset is identifiable. Since Partial Correlation, Sobol and Morris methods only determine influential parameters, care must be exercised if these parameter selection techniques are used to isolate identifiable parameters for model calibration.

\section{Conclusion}

In this paper, we examined parameter selection techniques based on global sensitivity analysis and compared the results to a local sensitivity-based method originally performed on the model (1). Four parameter selection techniques were applied to the HIV model (1) to determine the set of influential parameters. This process enables us to fix the noninfluential parameters and hence reduce the parameter dimensions for subsequent uncertainty quantification. We also showed that the accuracy of Sobol indices depends greatly on the model. In our HIV model, the second-order decomposition was not sufficiently accurate to represent the response. If one is interested in determining parameter identifiability issues, then it is recommended that one uses the parameter subset selection algorithm since it returns a set of identifiable parameters with smallest uncertainty.

It is important to note that there are several alternate choices for the model response. Our choice of the model response was motivated by the types of data that are available to us. However, one must carefully examine the cases when different model responses are chosen. It is important to remember that the parameters were determined influential in our analysis for our specific choice of model response. One idea for future work is to examine global sensitivity analysis using solely the T-cell counts as a response. Since the treatment attempts to increase the T-cell counts in patients, it is reasonable to focus on the T-cell counts alone. Similarly, one could focus on the viral loads $V_{I}+V_{N I}$ in an attempt to keep the viral loads low. Another aspect of analysis that we did not cover in this paper is to consider model response as a function of time. Recall that in our analysis we integrated the response in time to take into consideration of response at several time steps. In reality, we see that the states $T_{1}, T_{1}^{*}, \ldots, E_{2}$ can be mostly flat except for some jumps. Considering time-dependent model response will enable us to incorporate jumps that occur at certain times.

Finally, there are other methods of parameter selection that eliminate parameter identifiability issues. In particular, Active Subspace Methods detailed in 2, 11] determine a subspace of input parameter space which affects the response the most. This method does not isolate influential parameters from noninfluential parameters and the interpretation of the results may be more complicated. However, finding a linear combination of parameters that affects the response will resolve the parameter unidentifiability issues. Moreover, responses can be approximated based on the reduced parameter space, which is useful in subsequent model calibration and uncertainty quantification. Examining active subspace methods more closely as a part of parameter selection techniques will likely add more complete analysis on parameter selection techniques. 


\section{Acknowledgement}

The research of RCS and HTB was respectively supported in part by the Air Force Office of Scientific Research (AFOSR) through the grants AFOSR FA9550-11-1-0152 and AFOSR FA9550-12-1-0188.

\section{References}

[1] B. M. Adams, M. S. Ebeida, M. S. Eldred, J. D. Jakeman, L. P. Swiler, W. J. Bohnhoff, K. R. Dalbey, J. P. Eddy, K. T. Hu, D. M. Vigil, L. E. Bauman, and P. D. Hough. Dakota, a multilevel parallel object-oriented framework for design optimization, parameter estimation, uncertainty quantification, and sensitivity analysis: Version 5.3.1 user's manual. Sandia National Labs Report, No. SAND20102183, 2013.

[2] Y. Bang, H. S. Abdel-Khalik, and J. M. Hite. Hybrid reduced order modeling applied to nonlinear models. Internat. J. Numer. Methods Engrg., 91(9):929-949, 2012.

[3] H. T. Banks, R. Baraldi, K. Cross, K. Flores, C. McChesney, L. Poag, and E. Thorpe. Uncertainty Quantification in Modeling HIV Viral Mechanics. Technical report, CRSC-TR13-16, NC State University, Raleigh, NC, 2013. Journal of Theoretical Biology, submitted.

[4] H. T. Banks, A. Cintrón-Arias, and F. Kappel. Parameter selection methods in inverse problem formulation. In Mathematical modeling and validation in physiology, volume 2064 of Lecture Notes in Math., pages 43-73. Springer, Heidelberg, 2013.

[5] H. T. Banks, M. Davidian, S. Hu, G. M. Kepler, and E. S. Rosenberg. Modelling HIV immune response and validation with clinical data. J. Biol. Dyn., 2(4):357-385, 2008.

[6] H. T. Banks, S. L. Ernstberger, and S. L. Grove. Standard errors and confidence intervals in inverse problems: sensitivity and associated pitfalls. J. Inverse Ill-Posed Probl., 15(1):1-18, 2007.

[7] H. T. Banks and H. T. Tran. Mathematical and experimental modeling of physical and biological processes. Textbooks in Mathematics. CRC Press, Boca Raton, FL, 2009.

[8] F. Campolongo and A. Saltelli. Sensitivity analysis of an environmental model: an application of different analysis methods. Reliability Engineering 83 System Safety, 57(1):49-69, 1997. The Role of Sensitivity Analysis in the Corroboration of Models and its Links to Model Structural and Parametric Uncertainty.

[9] A. Cintrón-Arias, H. T. Banks, A. Capaldi, and A. L. Lloyd. A sensitivity matrix based methodology for inverse problem formulation. J. Inverse Ill-Posed Probl., 17(6):545-564, 2009.

[10] R. Confalonieri, G. Bellocchi, S. Bregaglio, M. Donatelli, and M. Acutis. Comparison of sensitivity analysis techniques: A case study with the rice model WARM. Ecological Modelling, 221(16):18971906, 2010.

[11] P. G. Constantine, E. Dow, and Q. Wang. Active subspace methods in theory and practice: applications to kriging surfaces. SIAM J. Sci. Comput., 36(4):A1500-A1524, 2014.

[12] M. J. W. Jansen. Analysis of variance designs for model output. Computer Physics Communications, $117(1-2): 35-43,1999$.

[13] G. Lillacci and M. Khammash. Parameter estimation and model selection in computational biology. PLoS Comput. Biol., 6(3):e1000696, 17, 2010.

[14] X. Ma and N. Zabaras. An adaptive high-dimensional stochastic model representation technique for the solution of stochastic partial differential equations. J. Comput. Phys., 229(10):3884-3915, 2010.

[15] M. D. Morris. Factorial sampling plans for preliminary computational experiments. Technometrics, 33(2):161-174, 1991. 
[16] H. Rabitz and Ö. F. Alış. General foundations of high-dimensional model representations. J. Math. Chem., 25(2-3):197-233, 1999.

[17] A. Saltelli. Making best use of model evaluations to compute sensitivity indices. Computer Physics Communications, 145(2):280-297, 2002.

[18] A. Saltelli, P. Annoni, I. Azzini, F. Campolongo, M. Ratto, and S. Tarantola. Variance based sensitivity analysis of model output. Design and estimator for the total sensitivity index. Comput. Phys. Comm., 181(2):259-270, 2010.

[19] A. Saltelli, M. Ratto, T. Andres, F. Campolongo, J. Cariboni, D. Gatelli, M. Saisana, and S. Tarantola. Global sensitivity analysis. The primer. John Wiley \& Sons, Ltd., Chichester, 2008.

[20] R. C. Smith. Uncertainty Quantification: Theory, Implementation and Applications. Society for Industrial and Applied Mathematics (SIAM), Philadelphia, PA, 2014.

[21] I. M. Sobol'. Global sensitivity indices for nonlinear mathematical models and their Monte Carlo estimates. Math. Comput. Simulation, 55(1-3):271-280, 2001. The Second IMACS Seminar on Monte Carlo Methods (Varna, 1999).

[22] I. M. Sobol', S. Tarantola, D. Gatelli, S. S. Kucherenko, and W. Mauntz. Estimating the approximation error when fixing unessential factors in global sensitivity analysis. Reliability Engineering 86 System Safety, 92(7):957-960, 2007.

[23] T. Sumner, E. Shephard, and I. D. L. Bogle. A methodology for global-sensitivity analysis of timedependent outputs in systems biology modelling. Journal of The Royal Society Interface, 9(74):21562166, 2012. 